\title{
Toward deformation densities for intramolecular interactions without radical reference states using the fragment, atom, localized, delocalized, and interatomic (FALDI) charge density decomposition scheme
}

\author{
Jurgens Hendrik de Lange, ${ }^{1}$ Ignacy Cukrowski ${ }^{1}$ \\ Correspondence to: Ignacy Cukrowski (E-mail: ignacy.cukrowski@up.ac.za) \\ ${ }^{1}$ Department of Chemistry, Faculty of Natural and Agricultural Sciences, University of Pretoria, Lynnwood Road, \\ Hatfield, Pretoria 0002, South Africa
}

\begin{abstract}
A novel approach for calculating deformation densities is presented, which enables to calculate the deformation density resulting from a change between two chemical states, typically conformers, without the need for unphysical (radical) fragments. The Fragment, Atom, Localized, Delocalized and Interatomic (FALDI) charge density decomposition scheme is introduced, which is applicable to static electron densities (FALDI-ED), conformational deformation densities (FALDI-DD) as well as orthodox fragment-based deformation densities. The formation of an intramolecular $\mathrm{NH} \cdots \mathrm{N}$ interaction in protonated ethylene diamine is used as a case study where the FALDI-based conformational deformation densities (with atomic or fragment resolution) are compared with an orthodox EDAbased approach. Atomic and fragment deformation densities revealed in real-space details (unavailable from EDAs schemes) that (i) pointed at the origin of density changes associated with the intramolecular $\mathrm{H}$-bond formation and (ii) fully support the IUPAC $\mathrm{H}$-bond representation. The FALDI scheme is equally applicable to intra- and intermolecular interactions.
\end{abstract}

\section{Introduction}

Recent years have seen a large resurgence in research investigating the chemical nature of many weak inter- and intramolecular interactions. Some of these interactions have been known to chemists for decades, but new theoretical evidence suggests that their chemical nature and properties might not be as clear as classical chemistry thought. Examples include the classically repulsive homopolar hydrogenhydrogen contacts, ${ }^{1-5}$ dihydrogen interactions, ${ }^{6-8}$ and the large number of different hydrogen bonds. ${ }^{9-13}$ On the other hand, investigations into the nature of known interactions have also led to the discovery of new interactions which might be of interest to chemists, such as halogen bonding, ${ }^{14-16}$ anion-anion interactions ${ }^{17}$ and chalcogen bonds. ${ }^{18}$ While many debates related to weak interactions are ongoing in the theoretical community (eg., the debates regarding the nature of hydrogen-hydrogen contacts ${ }^{19-27}$ ), research into these areas of interest have while many debates related to weak interactions are ongoing in the theoretical community (eg., the debates regarding the nature of hydrogen-hydrogen contacts ${ }^{19-}$ ${ }^{27}$ ), research into these areas of interest have revealed how underdeveloped our theoretical understanding of all chemical bonds still is. Specifically, despite many excellent treatises on the physical events on bond formation, ${ }^{28}$ there is still a scientific need to shed more light on the nature of many intramolecular interactions commonly found in experimental conditions, and how a network of 
intramolecular interactions contributes to the chemical properties and reactivity of a molecular system. To this end, many tools, techniques and methodologies have been developed over the last few decades which are able to probe specific aspects of intramolecular interactions. Many of these can be classified as decomposition and transformation of (i) the molecular energy, including FAMSEC, ${ }^{29}$ EDA, ${ }^{30} \mathrm{ETS}^{31}$ IQA, ${ }^{32}$ and $\mathrm{SAPT}^{33}$ or (ii) the molecular charge density distribution, including QTAIM, ${ }^{34} \mathrm{FMO}^{35}{ }^{35} \mathrm{NBO}^{36}$ $\mathrm{NCl}^{37}$ and NOCV. ${ }^{38}$ Some of these methods have been combined, such as ETS-NOCV, ${ }^{39}$ in order to give a holistic viewpoint towards the energy and electron density phenomena regarding inter- and intramolecular interactions. While many of these techniques provide clear insights with regards to intermolecular bonding, discussions involving intramolecular interactions are generally much more muddled. Hence, obtaining a better understanding of intramolecular bonding is a very desirable goal, due to the extreme prevalence of intramolecular interactions in many physical sciences.

Bader's Quantum Theory of Atoms in Molecules ${ }^{34}$ (QTAIM) and his concept of a bond path (BP) or atomic interaction line (AIL) have experienced significant success as a relatively inexpensive calculation to identify and classify inter- and intramolecular interactions. Many of his claims regarding the chemical significance of BPs have been questioned, ${ }^{25-27}$ leading to unorthodox interpretations of an AIL, ${ }^{40}$ improvements of QTAIM's charge decomposition scheme, including the Non-Covalent Interaction ${ }^{37}(\mathrm{NCl})$ approach as well as a proposal to change the Bader's nomenclature from a bond path to a line path. ${ }^{41}$ The interpretation of both QTAIM's AlLs and NCl's 'attractive regions of concentration', as well as the interpretation of most charge decomposition analyses, is inherent in one of Feynman's theorems, ${ }^{42}$ that the most attractive forces on atoms are observed when an influx of charge is seen in the interatomic region. It is generally accepted that an accumulation of electron density within the interatomic region of two or more atoms is indicative of an interaction with a predominantly bonding character; although it was shown that accumulation of density with accompanying an AlL is also observed when atoms are involved in a repulsive interaction even when a molecular system is at a true equilibrium state. ${ }^{27}$ Moreover, the manner in which an accumulation of electron density is measured differs significantly between different methods, leading to inconsistent interpretations and conclusions. For instance, we have previously shown that the measure of charge concentration (whether it be AlLs or regions of concentration as used by QTAIM and $\mathrm{NCl}$, respectively) cannot be used to consistently differentiate between IQA-defined attractive and repulsive intermolecular interactions in water dimers ${ }^{43}$ or intramolecular interactions in metal complexes. ${ }^{44}$

The question then arises as to which specific measure of charge concentration gives the correct description of the nature of an interaction. To this end, the simplest and clearest measure of an accumulation or depletion of electron density is the deformation density, also known as the difference density, $\Delta \rho(\mathbf{r})$. It is defined at any given coordinate $\mathbf{r}$ (Eq. 1 ) as the change in electron density in real space between the final ( $f$ in) and initial (or reference, ref) state,

$$
\Delta \rho(\mathbf{r})=\rho(\mathbf{r})-\rho^{0}(\mathbf{r})
$$

where

$$
\rho^{0}(\mathbf{r})=\sum_{i}^{M_{0}} \rho_{i}^{0}(\mathbf{r})
$$

describes the contribution at $\mathbf{r}$ to the fin state of density coming from an $i$ number of non-interacting promelecules $M_{0}$. In such approach, for the resulting $\Delta \rho(\mathbf{r})$ to be meaningful, it is necessary for the coordinate system to be identical in the fin as well as ref state of a molecular system. In other words, all 
nuclear positions in the fin system must be the same as in promolecular state, with the only difference that each promolecule contains a subset of the total number of nuclei. The result is such that the distance between each nuclear coordinate $\mathbf{R}_{i}$ and each coordinate $\mathbf{r}, d_{\mathrm{R}, \mathrm{r}}$ remains constant, but different electron distributions in the different states lead to the change in density between promolecules and a final molecule, $\Delta \rho(\mathbf{r})$.

The deformation density is an extremely valuable and easily-interpretable tool. Not only does $\Delta \rho(\mathbf{r})$ give the change (accumulation or depletion) of density within the bonding region of an interaction (in a manner directly in line with Feynman's theorem), but also the change in density outside of the bonding region, revealing the various polarizations and charge transfers resulting from the formation of an interaction. Moreover, $\Delta \rho(\mathbf{r})$ is often used in conjunction with the so-called interaction energy (or bond formation energy, defined as the energy difference between promolecular and molecular states). This is the central approach of many EDA schemes, such as the Extend Transition State coupled with Natural Orbitals for Chemical Valence (ETS-NOCV) approach, ${ }^{39}$ because visualizing and investigating electron density changes is a powerful tool in explaining bond formation energies.

Using $\Delta \rho(\mathbf{r})$ works well when intermolecular interactions are investigated because each separate molecule of a molecular system constitutes a well-defined and physical reference state. Unfortunately, suitable reference states are usually not available in the case of intramolecular interactions and it is necessary to fragment the molecule into one or more unphysical states (usually radicals). This is a significant drawback as, on reconstructing a molecule, formation of a covalent bond must unavoidably distort a picture related to the formation of a much weaker intramolecular bond/interaction. Nonetheless, due to the extreme usefulness of $\Delta \rho(\mathbf{r})$ and despite its limitations, ETS-NOCV and other theories based on $\Delta \rho(\mathbf{r})$ have been used to study intramolecular interactions and, with careful attention to the choice of reference state and final interpretation, have yielded some interesting results. ${ }^{45,46}$ Besides the fact that the use of unphysical reference states has been the cause for a few strong and critical arguments, ${ }^{47}$ it is obvious that any approach eliminating their need would be advantageous.

This paper is primarily focused on addressing the above limitations inherent to many charge decomposition schemes. To this effect, a novel approach to approximate the deformation density resulting from a conformational change involving physically and chemically sound states, typically conformers, is presented. We use a few concepts originating from the Domain Averaged Fermi Hole $(\mathrm{DAFH})^{48,49}$ method to decompose the electron density into Fragment, Atomic, Localized, Delocalized and Interatomic contributions, hence arriving at the FALDI charge decomposition scheme. These decomposition products are computed for each conformer. When individual changes are summed up, a FALDI-deformation density (DD) distribution in real space for a relevant $r e f \rightarrow$ fin structural change is obtained. Moreover, all components of the FALDI-DD distribution can be examined individually to gain an additional insight and aid interpretation of more complex systems. Applicability and usefulness of the FALDI-DD approach is demonstrated using two conformers of protonated ethylenediamine $\left(\mathrm{Hen}^{+}\right)$. The linear, higher energy conformer is used as a ref state. The formation of an intramolecular $\mathrm{H}$-bond in spontaneously formed fin state of $\mathrm{Hen}^{+}$is investigated by different modes of FALDI. Results obtained are compared with an orthodox $\Delta \rho(\mathbf{r})$ approach involving a number of partitioning schemes of the fin state of $\mathrm{Hen}^{+}$. We conclude this work by pointing at possible applications of the FALDI charge decomposition scheme as well as future extension(s) towards more accurate description of conformational deformation density distributions in real chemical systems. 


\section{Theoretical Development}

2.1. Framework for conformational deformation densities. For the deformation density resulting from a conformational change of a molecule (reference to final state, $r e f \rightarrow f i n$ ) to be calculated in real space, two significant and necessary modifications must be made to the basic approach described by Eq. 1: (i) molecular fragments in the ref state must be interacting to eliminate partitioning of a molecule to radical state fragments and (ii) the change in the coordinate system, caused by variation in nuclear distances and relative orientations on the $r e f \rightarrow$ fin structural change, must be accounted for. These modifications can both be addressed by using a suitable charge partitioning scheme in computing the density distributions of both ref and fin states into smaller components. Next, each component should be separately transformed to allow for a change in the coordinate system, while still be interacting in both ref and fin.

Many different density partitioning schemes satisfy a general expression

$\rho(\mathbf{r})=\sum_{i}^{M} \rho_{i}(\mathbf{r})$

where the molecular density is recovered by summing up contributions made by $M$ fragments or atoms. For instance, exhaustive and non-overlapping schemes, such as the partitioning of the molecular density into atomic basins $\Omega_{i}$ separated by zero-flux surfaces (as used in QTAIM ${ }^{34}$ ), can be used to decompose the density completely in a set of $M$ domains, $\Omega_{i}$, Eq. 4 :

$\rho_{i}(\mathbf{r})=\left\{\begin{array}{c}\rho(\mathbf{r}) \\ 0\end{array}\right\} \begin{aligned} & \mathbf{r} \in \Omega_{i} \\ & \mathbf{r} \notin \Omega_{i}\end{aligned}$.

It is important to note, however, that the shape and volume of each domain $\Omega_{i}$ changes from the ref to fin state. This results in regions of space for which the change in density cannot be uniquely attributed to a specific fragment, rendering a non-overlapping scheme, such as QTAIM, unsuitable for conformational deformation densities. From this follows that the desired density decomposition scheme in Eq. 3 needs to be based on molecular-wide distributions for each $\rho_{i}(\mathbf{r})$ and with $\rho_{i}(\mathbf{r}) \neq 0$ for all $\mathbf{r}$. In other words, whereas QTAIM partitions the molecular electron density into exclusive and rigid domains, with the electron density associated with an atom or fragment given as an average count of all the electrons found from the nucleus up to the domain surface, we need a scheme which rather gives the electron density associated with an atom as a distribution across the entire space. We have found that the Domain Averaged Fermi Hole (DAFH) analysis, first developed by Ponec, ${ }^{48,49}$ provides a density decomposition satisfying this requirement.

2.2. Domain Averaged Fermi Hole based density decomposition. DAFH analysis evolved around the concept of the exchange-correlation electron hole, which arises from the correlated movements of electrons

$$
C\left(\mathbf{r}_{1}, \mathbf{r}_{2}\right)=2 \rho_{2}\left(\mathbf{r}_{1}, \mathbf{r}_{2}\right)-\rho\left(\mathbf{r}_{1}\right) \rho\left(\mathbf{r}_{2}\right)
$$


where $C\left(\mathbf{r}_{1}, \mathbf{r}_{2}\right)$ is the correlation function, ${ }^{50}$ which relates the degree to which the pair density deviates from the completely uncorrelated product of first-order densities. The exchange-correlation electron hole function, located at $\mathbf{r}_{2}$ and evaluated at $\mathbf{r}_{1}$ is expressed in Eq. 6

$\rho^{\text {Hole }}\left(\mathbf{r}_{1} ; \mathbf{r}_{2}\right)=\rho\left(\mathbf{r}_{1}\right)-\rho^{\text {cond }}\left(\mathbf{r}_{1} ; \mathbf{r}_{2}\right)$

where

$\rho^{\text {cond }}\left(\mathbf{r}_{1} ; \mathbf{r}_{2}\right)=\frac{\rho_{2}\left(\mathbf{r}_{1}, \mathbf{r}_{2}\right)}{\rho\left(\mathbf{r}_{2}\right)}$

is the conditional probability of finding an electron at $\mathbf{r}_{1}$ if an electron is already present at $\mathbf{r}_{2}$. The electron hole function (Eq. 6) always integrates over the molecular system to -1 and is used to measure the degree to which the electron density is reduced (or rather, excluded) at $\mathbf{r}_{1}$ due to correlated movement with an electron at $\mathbf{r}_{2}$. By substituting Eqs. 6 and 7 into Eq. 5 one obtains

$$
C\left(\mathbf{r}_{1}, \mathbf{r}_{2}\right)=\rho_{2}\left(\mathbf{r}_{1}, \mathbf{r}_{2}\right)-\rho^{\text {Hole }}\left(\mathbf{r}_{1} ; \mathbf{r}_{2}\right) \rho\left(\mathbf{r}_{2}\right)
$$

giving the correlation function in terms of the electron hole weighted with the charge density at $\mathbf{r}_{2}$. Since the hole calculates the origin $\left(r_{2}\right)$ of the excluded electron (at $\left.r_{1}\right)$, plotting Eq. 8 by varying $r_{1}$ but keeping $\mathbf{r}_{2}$ constant is effectively plotting the pseudo-dynamic probability density distribution of a single electron at $\mathbf{r}_{2}{ }^{51}$

If we vary $\mathbf{r}_{1}$ over all space, it then gives the total, molecular-wide density distribution of the electrons which can be found in a volume element $d \mathbf{r}_{2}$. Finally, $\mathbf{r}_{2}$ can be averaged across a domain $\Omega_{i}$ (through integrating the correlation function through $d \mathbf{r}_{2}$ over a domain $\Omega_{i}$ ) in order to reduce the $2^{\text {nd }}$-order electron hole and correlation functions to pseudo-second order distributions,

$$
g_{i}\left(\mathbf{r}_{1}\right)=-\int_{\Omega_{i}} C\left(\mathbf{r}_{1}, \mathbf{r}_{2}\right) d \mathbf{r}_{2}
$$

which gives the DAFH quantity, $g_{i}(\mathbf{r})$ associated with domain $\Omega_{i}$. In principle, therefore, in the same way that the correlation function (Eq. 8), when plotted as a function of varying $r_{1}$ gives a probability distribution of an electron currently in $d \mathbf{r}_{2}$ (due to exchange-correlation effects), so does $g_{i}(\mathbf{r})$, when plotted as a function of varying $\mathbf{r}$, by giving a probability distribution of the electrons found on average in $\Omega_{i}$ across entire space. The $g_{i}(\mathbf{r})$ quantity, therefore, is of critical importance in this work, as it gives us the contribution made to any coordinate $r$ by electrons found on average in a domain, or, alternatively, the portion of the electron density at any coordinate $r$ associated with a specific domain $\Omega_{i}$. This concept is shown visually in Figure 1. Figure 1a shows the QTAIM-defined electron population for a carbon atom in ethane, represented as an isosurface, and completely contained within the atomic domain defined by a set of zero-flux surfaces. On average, 0.5809 electrons are found within the carbon atomic basin, which is calculated by integrating the electron density over only the basin. It is important to note that the isosurface shown in Figure 1a contains the electrons localized to C1's atomic basin as 
a)

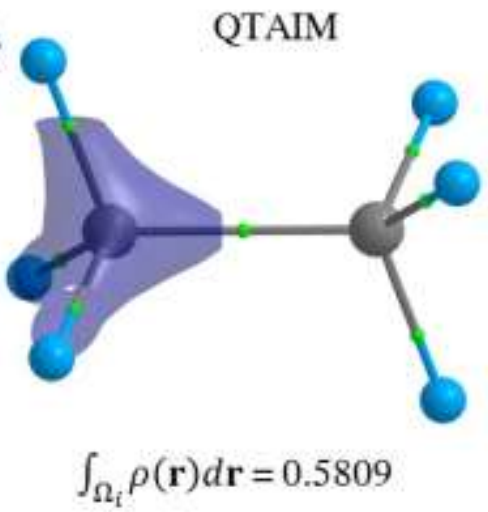

b)

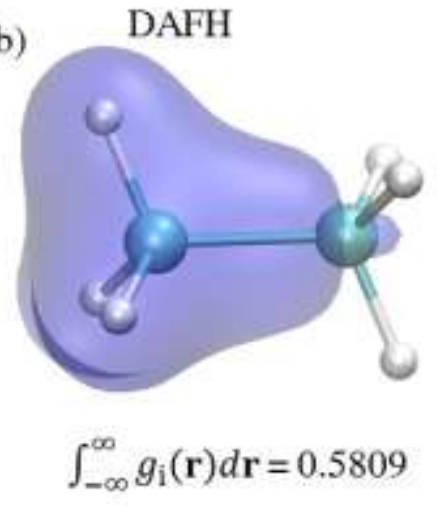

Figure 1. Average electron density associated with a carbon atom in ethane. a) QTAIM-defined electron population, and b) DAFH-defined electron population.

well as electrons delocalized to C1's basin from other basins. Correspondingly, the DAFH electron population (shown in Figure 1b) is identical to the QTAIM-defined population, but it is calculated by integrating $g_{i}(\mathbf{r})$ over the entire molecule and shows how the 0.5809 electrons are delocalized throughout the molecule. In other words, Figure $1 \mathrm{~b}$ displays the probability distribution of finding an electron anywhere in the molecule which can also be found, on average, in C1's atomic basin.

Although various domains $\Omega_{i}$ can be chosen, we have decided to use QTAIM atomic basins for the reasons stated by Bultinck et al. ${ }^{52}$ We also use the absolute values of $g_{i}(\mathbf{r})$, for practical reasons when dealing with the deformation density. In addition, we restrict ourselves to spin-independent functions, although it is not difficult to define spin-dependent variants of Eq. 9. ${ }^{51}$ Finally, to avoid the expensive integration of the correlation function over irregular QTAIM basins, we calculate $g_{i}(\mathbf{r})$ through comparison of the elements of various atomic overlap matrices (AOM)

$g_{i}\left(\mathbf{r}_{1}\right)=-\sum_{\lambda \sigma}^{N} \varphi_{\lambda}\left(\mathbf{r}_{1}\right) \varphi_{\sigma}\left(\mathbf{r}_{1}\right) S_{\sigma \lambda}^{\Omega}$

where

$S_{\sigma \lambda}^{\Omega_{i}}=\left\langle\varphi_{\sigma} \mid \varphi_{\lambda}\right\rangle_{\Omega}=\int_{\Omega_{i}} \varphi_{\sigma}\left(\mathbf{r}_{1}\right) \varphi_{\lambda}\left(\mathbf{r}_{1}\right) d \mathbf{r}_{1}$

and $S_{\sigma \lambda}^{\Omega_{i}}$ stands for elements of the AOM associated with a specific domain $\Omega_{i}$. In single determinant wavefunctions, using the elements of all AOM's related to each domain, $g_{i}(\mathbf{r})$ is calculated exactly and corresponds to the actual correlation function. However, for more correlated wavefunctions, this does not hold true anymore and it is either necessary to calculate and integrate the exact correlation function in Eq. 5 or approach Coulomb correlation in an approximate manner. We have opted for the latter option through the use of the Müller approximation by linearly expanding the first-order density matrix with partial occupation numbers. ${ }^{53}$ However, we note that multiple methods have been suggested for more accurate and efficient treating of Coulomb correlation in DAFH analyses, and it is something we will incorporate in our own scheme in the future. 
In traditional DAFH analyses, $g_{i}(\mathbf{r})$ is expressed as a matrix equation that is diagonalized in order to decompose $g_{i}(\mathbf{r})$ into $N$ one-electron functions. Such an approach is counter-productive when the conformational deformation density is concerned, as the relative order of the one-electron DAFH functions might change within a matrix when the $r e f \rightarrow$ fin structural change takes place, creating additional difficulties. Furthermore, in this work we are more interested in the concept of $g_{i}(\mathbf{r})$, which we take from DAFH, rather than the full natural orbital approach.

2.3. General properties of the DAFH. It is important to point at a number of properties of $g_{i}(\mathbf{r})$ that are relevant to constructing conformational deformation density distributions:

1. $g_{i}(\mathbf{r})$ is a complete decomposition of the molecular electron density, at any point $\mathbf{r}$, into contributions from all $M$ domains, as shown in Eq. 12,

$\rho(\mathbf{r})=\sum_{i}^{M} g_{i}(\mathbf{r})$

$g_{i}(\mathbf{r})$ therefore satisfies Eq. 3, but is not limited by the restriction in Eq. 4; hence, it is well-suited for studying the change of density associated with a specific domain.

2. Integration of $g_{i}(\mathbf{r})$ over the entire molecular space yields the associated average density within a specific domain $\Omega_{i}$ :

$$
N\left(\Omega_{i}\right)=\int_{\Omega_{i}} \rho(\mathbf{r}) d \mathbf{r}=\int_{-\infty}^{\infty} g_{i}(\mathbf{r}) d \mathbf{r}
$$

where $N\left(\Omega_{i}\right)$ is the QTAIM-defined electron population of the domain. Integrating the electron density over the volume of $\Omega_{i}$, or integrating $g_{i}(\mathbf{r})$ over the entire molecular system gives the same result. In this regard, the change in the atomic electron population from a ref to a fin conformer, $\Delta N\left(\Omega_{i}\right)$, is recovered whether $\rho(\mathbf{r})$ or $g_{i}(\mathbf{r})$ is used. Importantly, however, using $g_{i}(\mathbf{r})$ to calculate deformation density does not depend on changing domain shapes and volumes.

3. Integration of $g_{i}(\mathbf{r})$ over only a single domain $\Omega_{i}$ leads to the often used localization index, (LI), Eq. 14

$$
\lambda\left(\Omega_{i}\right)=\int_{\Omega_{i}} g_{i}(\mathbf{r}) d \mathbf{r}
$$

giving the number of electrons localized to a single atomic basin, whereas integration over any other domain $\Omega_{j}$ leads to the halved delocalization index (DI), Eq. 15,

$$
\delta\left(\Omega_{i}, \Omega_{j}\right)=\int_{\Omega_{j}} g_{i}\left(\mathbf{r}_{1}\right) d \mathbf{r}_{1}+\int_{\Omega_{i}} g_{j}\left(\mathbf{r}_{2}\right) d \mathbf{r}_{2}=2 \int_{\Omega_{j}} g_{i}\left(\mathbf{r}_{1}\right) d \mathbf{r}_{1}
$$


giving the number of electrons delocalized between two atomic basins. The $\mathrm{LI}$ and $\mathrm{DI}$ are related to the electron population of domain $\Omega_{i}$ through

$$
N\left(\Omega_{i}\right)=\lambda\left(\Omega_{i}\right)+\sum_{X \neq i}^{M} \frac{1}{2} \delta\left(\Omega_{i}, \Omega_{X}\right) .
$$

Note that the contribution which $g_{i}(\mathbf{r})$ makes at $\mathbf{r}$ (regardless if it is towards the localized or delocalized density of $\Omega_{i}$ ) depends only on whether $r \in \Omega_{i}$. Hence, Eq. 16 can be rewritten in terms of $g_{i}(\mathbf{r})$ as:

$$
N\left(\Omega_{i}\right)=\int_{\Omega_{i}} g_{i}(\mathbf{r}) d \mathbf{r}+\int_{\Omega_{j}} g_{i}(\mathbf{r}) d \mathbf{r}
$$

4. The distribution of $g_{i}(\mathbf{r})$ can be interpreted as the probability of finding any one of $N\left(\Omega_{i}\right)$ electrons (contained on average in $\Omega_{i}$ ), at any $\mathbf{r}$. Also, $g_{i}(\mathbf{r})$ can be visualized as the distribution of the electrons of $\Omega_{i}$ over the molecular system through electron delocalization (exchange-correlation) effects. If $g_{i}(\mathbf{r})=0$, then none of the electrons in $\Omega_{i}$ can be found at $\mathbf{r}$ and if all $\mathbf{r} \notin \Omega_{i}$, then the electrons of $\Omega_{i}$ are fully localized and $\rho(\mathbf{r})=g_{i}(\mathbf{r})$ for $\left.\mathbf{r} \in \Omega_{i}\right)$.

2.4 Introducing the FALDI density decomposition scheme. The properties of $g_{i}(\mathbf{r})$ make it ideal for the calculation of conformational deformation densities. This is because density at any coordinate $r$ in a final molecular state can be decomposed into atomic contributions and compared to relevant $g_{i}(\mathbf{r})$ at transformed $\mathbf{r}^{\prime}=\mathbf{A}_{i} \mathbf{r}$ in a reference state,

$$
\Delta_{c} \rho(\mathbf{r})={ }^{f i n} \rho(\mathbf{r})-{ }^{r e f} \rho\left(\mathbf{A}_{i} \mathbf{r}\right)=\sum_{i}^{M}{ }^{f i n} g_{i}(\mathbf{r})-\sum_{i}^{M}{ }^{r e f} g_{i}\left(\mathbf{A}_{i} \mathbf{r}\right)
$$

where we have introduced $\Delta_{c} \rho(\mathbf{r})$ to signify the conformational deformation density, $M$ is the total number of atoms and $\mathbf{A}_{i}$ is the relevant transformation matrix relating the translation and rotation of the $i$ th atom's coordinates in the fin state to the ref state. The difference between each ${ }^{f i n} g_{i}(\mathbf{r})$ and ${ }^{r e f} g_{i}\left(\mathbf{A}_{i} \mathbf{r}\right)$ pair results in the deformation density associated with a specific atom (or domain) $\Omega_{i}$, and hence we introduce here the first two terms in our FALDI decomposition scheme, an Atomic Electron Density (AED) distribution, defined as the static $g_{i}(\mathbf{r})$ for the ith atom in a conformer, and when used in the deformation density context, an Atomic Deformation Density (ADD) distribution, defined for the ith atom as ${ }^{\text {fin }} g_{i}(\mathbf{r})-{ }^{r e f} g_{i}\left(\mathbf{A}_{i} \mathbf{r}\right)$ from two states.

The sum of various AED and ADD distributions gives rise to the next two terms in our FALDI decomposition scheme, a Fragment Electron Density (FED) distribution, defined as the sum of $g_{i}(\mathbf{r})$ for any number of atoms forming a molecular fragment, and a Fragment Deformation Density (FDD) distribution, defined as the sum of $\left\{{ }^{f i n} g_{i}(\mathbf{r})-{ }^{r e f} g_{i}\left(\mathbf{A}_{i} \mathbf{r}\right)\right\}$ changes resulting from two states. The sum of FDD distributions computed for all fragments a molecule is made of (or the sum of all ADD distributions) gives the total (molecular) deformation density (TDD) distribution $\Delta_{c} \rho(\mathbf{r})$ defined in Eq. 18. We want 
to stress again that ADD and FDD distributions require separate transformation matrix $\left(\mathbf{A}_{i} \mathbf{r}\right)$ for each atomic basin $\Omega_{i}$ as this is critical for the calculation of $\Delta_{c} \rho(\mathbf{r})$. Finally, they are very useful separate analysis tools in exploring the origin of the total change in density between two states at either atomic or fragment level.

2.5. Decomposing AED distributions into localized, delocalized and interatomic contributions. The conformational deformation density calculated by Eq. 18 can be difficult to interpret for certain systems and molecular transformations. The reason for this is the manner through which density shared between two atomic basins is transformed from the ref to the fin conformer: density delocalized between two atomic basins $\left(\Omega_{i}\right.$ and $\left.\Omega_{j}\right)$ with different transformation matrices $\left(\mathbf{A}_{i}\right.$ and $\left.\mathbf{A}_{j}\right)$ should be uniquely and separately transformed in order to take into account its dependence on two transformation matrices, as opposed to density localized to one atom (which only depends on $\mathbf{A}_{i}$ ). As it stands though, $\Delta_{c} \rho(\mathbf{r})$ calculated through Eq. 18 presents the interatomic delocalized density simultaneously as half transformed via the transformation matrix of one atom $\left(\mathbf{A}_{i}\right)$ and half through the transformation matrix of the other atom $\left(\mathbf{A}_{j}\right)$. As an example of this problem, consider a system with two atoms, $A$ and $B$, where $A$ is transformed from ref to fin via $\mathbf{A}_{A}$ and $B$ remains untransformed $\left(\mathbf{A}_{B}=\mathbf{1}\right)$. The density localized only to the basin of $A$ in ref is correctly transformed through $\mathbf{A}_{A}$ and compared with the density localized to the basin of $A$ in fin. However, half of the density delocalized between $A$ and $B$ in ref is transformed relative to $A$ through $\mathbf{A}_{A}$ and compared to the interatomic delocalized density in fin, resulting in an apparent density change as if $B$ was also transformed through $\mathbf{A}_{A}$. Correspondingly, half of the density delocalized between $A$ and $B$ in ref remains untransformed, and results in an apparent density change as if $A$ remained untransformed. If another atom $C$ is transformed in the same manner as $A$ (with $\mathbf{A}_{A}=\mathbf{A}_{C}$, if $A$ and $C$ are parts of the same fragment) then the density shared by $A$ and $C$ is correctly transformed from ref to fin, but not so the density shared between $C$ and $B$. This problem of density delocalized across atoms with different transformation matrices appears as apparent artefacts on the TDD distribution, and although $\Delta_{c} \rho(\mathbf{r})$ can still be interpreted correctly with some care, it becomes very difficult with anything but the simplest molecules. Note that the apparent artefacts in $\Delta_{c} \rho(\mathbf{r})$ are self-eliminating in that $\Delta_{c} \rho(\mathbf{r})$ integrated over all space is 0 because regions where the delocalized density apparently increases due to the problem described above is cancelled out by regions where the delocalized density apparently decreases.

We present next an approximation to the way in which $\Delta_{c} \rho(\mathbf{r})$ is calculated, which greatly aids the interpretation of $\Delta_{c} \rho(\mathbf{r})$ whilst keeping $\Delta_{c} \rho(\mathbf{r})$ integrated over all space to 0 . Since it is only the density shared between atomic basins which is affected by incorrect transformation, we first develop expressions for decomposing the static density into localized and delocalized contributions in real space. These expressions form the final terms of our FALDI density decomposition. We then develop an approximate method for transforming delocalized density distributions from ref to fin through multiple transformation matrices.

The total static electron density can be expanded algebraically and in terms of $g_{i}(\mathbf{r})$ it can be expressed as

$$
\rho(\mathbf{r})=\sum_{i}^{M} g_{i}(\mathbf{r}) g_{i}(\mathbf{r}) \rho^{-1}(\mathbf{r})+2 \sum_{i}^{M-1} \sum_{j=i+1}^{M} g_{i}(\mathbf{r}) g_{j}(\mathbf{r}) \rho^{-1}(\mathbf{r})
$$


and, accordingly, the $g_{i}(\mathbf{r})$ distribution of each atom can be decomposed by

$$
g_{i}(\mathbf{r})=g_{i}(\mathbf{r}) g_{i}(\mathbf{r}) \rho^{-1}(\mathbf{r})+\sum_{X \neq i}^{M} g_{i}(\mathbf{r}) g_{X}(\mathbf{r}) \rho^{-1}(\mathbf{r})=\lambda_{i}(\mathbf{r})+\sum_{X \neq i}^{M} \delta_{i, X}(\mathbf{r})
$$

This approximation calculates the fraction of the electron density which any atom contributes at $\mathbf{r}$, weighted with either the same atom's contribution (as in the first term in Eq. 20) or another atom's contribution (the second term in Eq. 20). The result is a full decomposition of the electron density (Eq. 19) or the atomic density contribution (Eq. 20) at a specific coordinate $r$ in terms of density localized to an atom $\left(g_{i}(\mathbf{r}) g_{i}(\mathbf{r}) \rho^{-1}(\mathbf{r})\right)$ and density delocalized between an atom and all other atoms $\left(\sum_{X \neq i}^{M} g_{i}(\mathbf{r}) g_{X}(\mathbf{r}) \rho^{-1}(\mathbf{r})\right)$. We have used the same symbols commonly employed in QTAIM methodology for localization indices $\left(\lambda\left(\Omega_{i}\right)\right)$ or delocalization indices $\left(\delta\left(\Omega_{i}, \Omega_{\mathrm{x}}\right)\right)$ in order to imply the same meaning, although mathematically $\lambda_{i}(\mathbf{r})$ and $\delta_{i, X}(\mathbf{r})$ are not directly equivalent to $\lambda\left(\Omega_{i}\right)$ and $\delta\left(\Omega_{i}, \Omega_{X}\right)$. These two terms, $\lambda_{i}(\mathbf{r})$ and $\delta_{i, X}(\mathbf{r})$, are known in our FALDI decomposition scheme as Localized and Delocalized Electron Density (LED and DED) distributions, respectively. The $\delta_{i, X}(\mathbf{r})$ term is defined for a domain $\Omega_{i}$ together with all other domains $\Omega_{X}$; for the special case of only two atoms, we specify $\delta_{i, j}(\mathbf{r})$ and introduce our final FALDI term, Interatomic Electron Density (IED) distributions.

LED distributions give the probability of finding an electron at $r$ which is strongly localized, in an exchange-correlation sense, to a domain $\Omega_{i}$. While the probability of finding such electrons are nonzero even when $\mathbf{r}$ is outside a domain $\Omega_{i}\left(\mathbf{r} \notin \Omega_{i}\right)$, LED distributions tend to be much more concentrated in the domain they are associated with. The change in $\lambda_{i}(\mathbf{r})$ for two different states, $\Delta \lambda_{i}(\mathbf{r})={ }^{\text {fin }} \lambda_{i}(\mathbf{r})-{ }^{r e f} \lambda_{i}\left(\mathbf{A}_{i} \mathbf{r}\right)$, gives the deformation of the density localized to an atom, which we call a Localized Deformation Density (LDD) distribution.

In case of IED distributions, they give the probability for an electron at $\mathbf{r}$ to be delocalized between two domains $\Omega_{i}$ and $\Omega_{j}$, or in the case of DED distributions, for all pairs of $\Omega_{i}$ with all other domains $\Omega_{x}$. In other words, the IED gives the real space distribution of electrons shared by two basins, and shows very interesting and different patterns depending on the nature of the interaction. As an example, the IED distribution of a covalent $\mathrm{C}-\mathrm{C}$ bond in ethane is compared to the IED distribution of a van der Waals (vdW) interaction between $\mathrm{O} \cdots \mathrm{H}$ in a water dimer in Figure 2, where the vdW interaction displays a totally different topology than the covalent bond; this clearly illustrates how electrons are shared between two atoms in real space. 


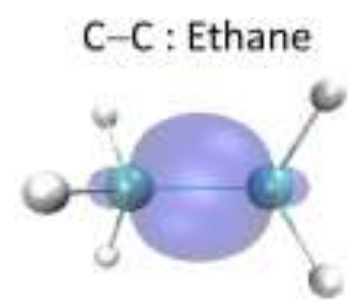

\section{O...H: Water dimer}

\section{Interaction electron density (IED): $\kappa_{i_{j}}(\mathbf{r})$}
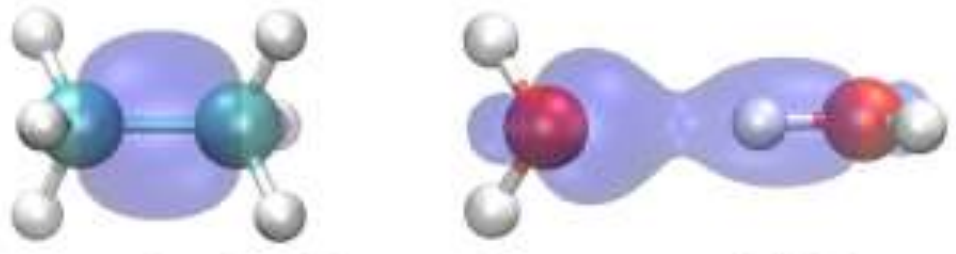

\section{Delocalized electron density $\quad \delta_{i, j}(\mathbf{r})$ (DED):}

Figure 2. Interatomic-ED and delocalized-ED distributions for a $C-C$ bond in ethane, isovalue $=0.01$ au, and an $\mathrm{O} \cdots \mathrm{H}$ interaction in a water dimer, isovalue $=0.001 \mathrm{au}$.

As mentioned at the beginning of this section, the localized density of an atom (a LED distribution) in the ref state is easily transformed via $\mathbf{A}_{\mathbf{i}} \mathbf{r}$ and compared to the fin state to generate a LDD distribution. On the other hand, interatomic delocalized density (an IED or DED distribution) needs to be transformed using two transformation matrices simultaneously in order to calculate Interatomic and Delocalized Deformation Density (IDD and DDD, respectively) distributions. While there are a number of different ways to approach this problem, we have opted to use a relatively simple scaling scheme based on projections of the internuclear vector to generate $\mathbf{r}^{\prime}$ from the transformed coordinates of domains $\Omega_{i}$ and $\Omega_{j}, \mathbf{r}_{i}^{\prime}$ and $\mathbf{r}_{j}^{\prime}$. The derivation of this approach is discussed below.

Given the transformations for $\mathbf{r}$ to the relevant $\mathbf{r}_{i}^{\prime}$ and $\mathbf{r}_{j}^{\prime}$ of two atomic basins $\Omega_{i}$ and $\Omega_{j}$,

$$
\begin{aligned}
& \mathbf{r}_{i}^{\prime}=\mathbf{A}_{i} \mathbf{r} \\
& \mathbf{r}_{j}^{\prime}=\mathbf{A}_{j} \mathbf{r}
\end{aligned}
$$

we can calculate the value of $\delta_{i, j}(\mathbf{r})$ in the ref state at $\mathbf{r}$ as a weighted combination of $\delta_{i, j}$ at $\mathbf{r}_{i}{ }_{i}$ and $\mathbf{r}^{\prime}{ }_{j}$, as shown in Eq. 22,

$$
{ }^{r e f} \delta_{i, j}^{\prime}(\mathbf{r})=w_{i}(\mathbf{r})^{r e f} \delta_{i, j}\left(\mathbf{r}_{i}^{\prime}\right)+w_{j}(\mathbf{r})^{r e f} \delta_{i, j}\left(\mathbf{r}_{j}^{\prime}\right)=w_{i j}(\mathbf{r})^{r e f} \delta_{i, j}\left(\mathbf{r}_{i}^{\prime}\right)+\left[1-w_{i j}(\mathbf{r})\right]^{r e f} \delta_{i, j}\left(\mathbf{r}_{j}^{\prime}\right)
$$

The weighting factors $w_{i j}$ can be generated, Eq. 23 , by projecting the vector from the nuclear coordinate of the $i$ th atom $\mathbf{R}_{i}$ to $\mathbf{r}, \mathbf{r}-\mathbf{R}_{i}$, onto the internuclear vector, $\mathbf{R}_{i j}$, and calculating the fraction along $\mathbf{R}_{i j}$ where $\mathbf{r}$ is projected,

$$
w_{i j}(\mathbf{r})=1-\frac{\left(\mathbf{r}-\mathbf{R}_{i}\right) \cdot \mathbf{R}_{i j}}{\left|\mathbf{R}_{i j}\right|^{2}}
$$


For coordinates $\mathbf{r}$ between $\mathbf{R}_{i}$ and $\mathbf{R}_{j},{ }^{r e f} \delta_{i, j}^{\prime}(\mathbf{r})$ can be calculated as a weighted combination of ${ }^{r e f} \delta_{i, j}(\mathbf{r})$ in terms of $\mathbf{r}_{i}^{\prime}$ and $\mathbf{r}_{j}^{\prime}$, whereas outside of $\mathbf{R}_{i}$ and $\mathbf{R}_{j}{ }^{r e f} \delta_{i, j}^{\prime}(\mathbf{r})$ depends only on $\mathbf{r}_{i}^{\prime}$ or $\mathbf{r}_{j}^{\prime}$ :

$$
{ }^{r e f} \delta_{i, j}^{\prime}(\mathbf{r})=\left\{\begin{array}{c}
w_{i j}(\mathbf{r})^{r e f} \delta_{i, j}\left(\mathbf{r}_{i}^{\prime}\right)+\left[1-w_{i j}(\mathbf{r})\right]^{r e f} \delta_{i, j}\left(\mathbf{r}_{j}^{\prime}\right) \\
{ }^{r e f} \delta_{i, j}\left(\mathbf{r}_{i}^{\prime}\right) \\
{ }^{r e f} \delta_{i, j}\left(\mathbf{r}_{j}^{\prime}\right)
\end{array}\right\} \begin{gathered}
0<w_{i j}(\mathbf{r})<1 \\
w_{i j}(\mathbf{r}) \geq 1 \\
w_{i j}(\mathbf{r}) \leq 0
\end{gathered}
$$

This approximation lets us calculate ${ }^{r e f} \delta_{i, j}^{\prime}(\mathbf{r})$ (where the prime indicates the use of the approximation) at $\mathbf{r}$, and compare directly to ${ }^{\text {fin }} \delta_{i, j}(\mathbf{r})$, which gives the change in interatomic or interfragment density contributions resulting from two states. This protocol generates an approximated IDD distribution and the sum of all IDD distributions associated with a specific atom gives its DDD distribution. In the following final section, we will put all of the FALDI terms together to form expressions for calculating conformational deformation densities.

2.6 Conformational Deformation Densities using the FALDI-DD decomposition. The total conformational deformation density, using the approximation for ${ }^{r e f} \delta_{i, j}^{\prime}(\mathbf{r})$, is then

$$
\Delta_{c} \rho(\mathbf{r})=\Delta_{c} \lambda(\mathbf{r})+\Delta_{c} \delta(\mathbf{r})
$$

where

$$
\Delta_{c} \lambda(\mathbf{r})=\sum_{i}^{M}\left[{ }^{f i n} \lambda_{i}(\mathbf{r})-{ }^{r e f} \lambda_{i}\left(\mathbf{A}_{i} \mathbf{r}\right)\right]
$$

gives the total localized density change for each atomic basin $\Omega_{i}$ (a LDD distribution), and

$$
\Delta_{c} \delta(\mathbf{r})=2 \sum_{i}^{M-1} \sum_{j=i+1}^{M}\left[{ }^{f i n} \delta_{i, j}(\mathbf{r})-{ }^{r e f} \delta_{i, j}^{\prime}(\mathbf{r})\right]
$$

gives the total delocalized density change for each unique atomic pair $\Omega_{i}$ and $\Omega_{j}$ (an IDD distribution). Note that the sum of all IDD distributions associated with atom $\Omega_{i}$ gives the DDD distribution of atom $\Omega_{i}$

$$
\Delta_{c} \delta_{i, X}(\mathbf{r})=\sum_{j \neq i}^{M} \Delta_{c} \delta_{i, j}(\mathbf{r})
$$

and the sum of the DDD and LDD distributions of atom $\Omega_{i}$ gives its ADD distribution

$$
\Delta_{c} g_{i}(\mathbf{r})=\Delta_{c} \lambda_{i}(\mathbf{r})+\Delta_{c} \delta_{i, X}(\mathbf{r}) .
$$

The sum of all ADD distributions for atoms within a fragment $\{F\}$ gives its FDD distribution 


$$
\Delta_{c} g^{\{F\}}(\mathbf{r})=\sum_{i}^{M_{\{F\}}} \Delta_{c} g_{i}(\mathbf{r}) .
$$

where $M_{\{F\}}$ is the total number of fragments.

Each FDD distribution can also be decomposed into the density localized to a fragment, as well as the density delocalized between different fragments

$$
\Delta_{c} g^{\{F\}}(\mathbf{r})=\Delta_{c} \lambda^{\{F\}}(\mathbf{r})+\Delta_{c} \delta^{\{F\}}(\mathbf{r})
$$

where

$\Delta_{c} \lambda^{\{F\}}(\mathbf{r})=\sum_{i}^{M_{\{F\}}} \Delta_{c} \lambda_{i}(\mathbf{r})+2 \sum_{i}^{M_{\{F\}}-1} \sum_{j=i+1}^{M_{\{F\}}} \Delta_{c} \delta_{i, j}(\mathbf{r})$

and

$$
\Delta_{c} \delta^{\{F\}}(\mathbf{r})=\sum_{i}^{M_{\{F\}}} \sum_{k}^{M} \Delta_{c} \delta_{i, k}(\mathbf{r})
$$

where $k \notin\{F\}$.

Finally, the sum of all $M$ FDD distributions gives again the conformational deformation density, as defined in Eq. 25

$$
\Delta_{c} \rho(\mathbf{r})=\sum_{i}^{\{M\}} \Delta_{c} g^{\{i\}}(\mathbf{r})
$$

where $\{M\}$ is the total number of fragments.

Scheme 1 shows the full decomposition scheme. With regards to our proposed nomenclature, we will use (1) FALDI-ED, where ED can stand for either FED, AED, LED, DED or IED to describe the FALDI decomposition when it is applied to the static electron density distribution of a single molecule, and (2) FALDI-DD, where DD can stand for either FDD, ADD, LDD, DDD and IDD to describe the FALDI decomposition when it is applied to the change in density arising from a conformational change.

Lastly, we wish to note that the same decomposition is applicable to an orthodox fragmentation of the molecular system that involves promolecular deformation densities by setting ${ }^{r e f} \delta_{i, j}(\mathbf{r})=0$, $\Delta \delta_{i, j}(\mathbf{r})={ }^{f i n} \delta_{i, j}(\mathbf{r})$ and $\mathbf{A}_{i}=\mathbf{1}$ for all $i$. The FALDI-DD scheme can therefore be used to interpret and analyse orthodox deformation densities, as well as compare various FALDI-DD terms of both orthodox and conformational deformation densities. Hence, our decomposition scheme can be used in conjunction with the very useful natural orbital expansion of the various components of $\Delta \rho(\mathbf{r}$ ) (as is implemented in ETS-NOCV), in order to provide additional insight to the changes occurring during bond formation. 


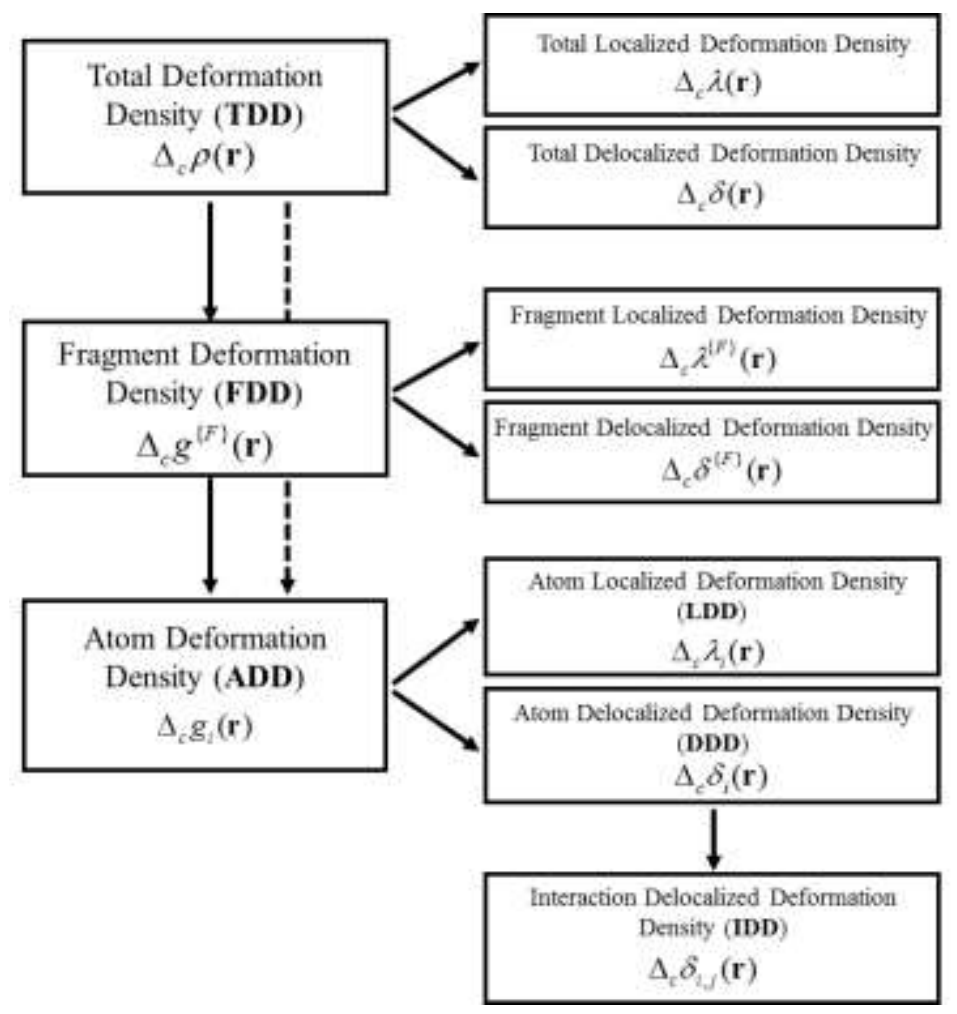

Scheme 1. Proposed FALDI-DD decomposition of the deformation density.

\section{Computational details}

All systems were optimized in Gaussian 09, rev.D, ${ }^{54}$ using RB3LYP-D3/6-311++G(d,p) in gas-phase. AIMAll version $16.05 .18^{55}$ was used to calculate atomic overlap matrices for all systems, using suitable wavefunction files generated by Gaussian and at the same level of theory and basis set. Atomic overlap matrices were used to perform the FALDI decomposition and conformational deformation densities using in-house software. All FALDI isosurfaces were visualized using VMD. ${ }^{56}$ Orthodox deformation density calculations were performed and visualized with ADF 2014, ${ }^{57}$ using RB3LYP-D3/ATZP; however, similar calculations were also performed in Gaussian and visualized with VMD for comparison. Gaussian coordinates for all optimized molecules as well as promolecules are included in Section S1 of the Supplementary Information.

\section{Results and Discussion}

Our focus is on gaining an insight on the density changes associated with the formation of an intramolecular interaction from the FALDI perspective and, as a case study, we will analyse a classical intramolecular $\mathrm{H}$-bond in the lowest energy conformer of protonated ethylenediamine $\left(\mathrm{Hen}^{+}\right)$used here as a fin state of $\mathrm{Hen}^{+}$. The $\mathrm{N} 8-\mathrm{H} 11 \cdots \mathrm{N} 7$ bond (Figure 3) with $\mathrm{d}(\mathrm{N} 7, \mathrm{H} 11)=2.110 \AA$, is formed spontaneously and, relative to a linear conformer, used here as a ref state, the molecular energy decreases, e.g., by $-4.1 \mathrm{kcal} \cdot \mathrm{mol}^{-1}$ at the $\mathrm{MP} 2 / 6311++\mathrm{G}(\mathrm{d}, \mathrm{p})$ in aqueous solution. In sections that follow we will perform a brief comparative analysis between an orthodox approach, using various fragmentation schemes, and the FALDI method reported in this work. Furthermore, we will demonstrate 
some unique features of FALDI by exploring deformation densities resulting from a ref $\rightarrow$ fin structural change with a main focus on the $\mathrm{H}_{2} \mathrm{~N} 8-\mathrm{H} 11 \cdots \mathrm{N}_{7} \mathrm{H}_{2}-\mathrm{C} 1$ region.

4.1. Total deformation density from orthodox $\Delta \rho(r)$ and FALDI $\Delta \rho_{c}(r)$. An orthodox decomposition approach requires partitioning of a molecule to promolecules (or fragments). Four partitioning schemes were tested here where (i) $\mathrm{Hen}^{+}$was cut in the middle, which resulted in two, $-\mathrm{CH}_{2} \mathrm{NH}_{2}$ and $-\mathrm{CH}_{2} \mathrm{NH}_{3}{ }^{+}$ fragments (2 $F_{1}$ scheme), (ii) $\mathrm{NH}_{2}$ group was cut off, hence $-\mathrm{NH}_{2}$ and $-\mathrm{CH}_{2} \mathrm{CH}_{2} \mathrm{NH}_{3}{ }^{+}$fragments were formed ( $2 \mathrm{~F}_{2}$ scheme), (iii) $\mathrm{NH}_{3}{ }^{+}$group was cut off resulting in two, $-\mathrm{NH}_{3}{ }^{+}$and $-\mathrm{CH}_{2} \mathrm{CH}_{2} \mathrm{NH}_{2}$ fragments $\left(2 \mathrm{~F}_{3}\right.$ scheme) and (iv) both terminal groups were cut off generating three, $-\mathrm{NH}_{2},-\mathrm{CH}_{2} \mathrm{CH}_{2}-$ and $-\mathrm{NH}_{3}{ }^{+}$ fragments ( $3 F$ scheme) (this is pictorially presented in Scheme S1 in the Supplementary Information).

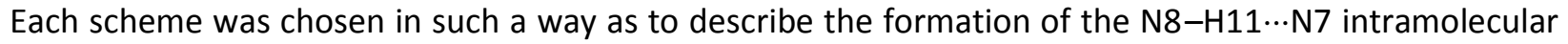
interaction.

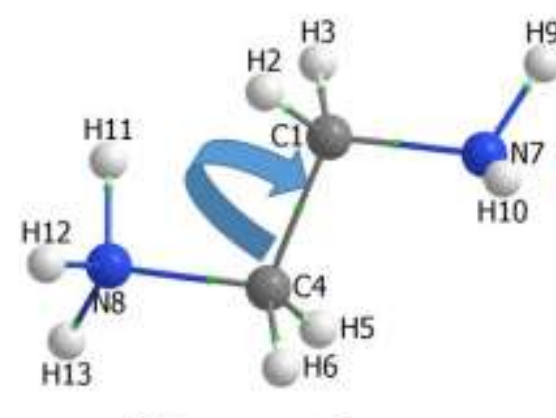

ref: linear conformer

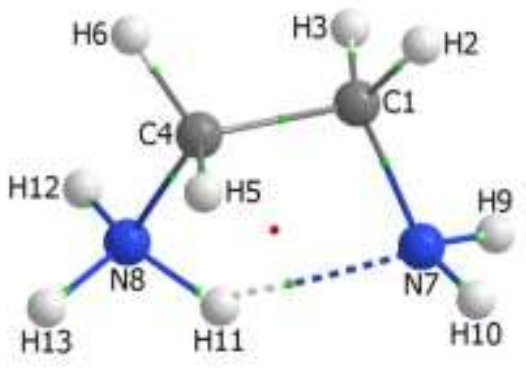

fin: equilibrium conformer

Figure 3. Molecular graphs of linear $\mathrm{Hen}^{+}$(ref) and equilibrium $\mathrm{Hen}^{+}$(fin) conformers for the calculation of conformational deformation densities using FALDI. The ref conformer is rotated around the N7,C1,C4,N8 dihedral angle. Atoms' numbering is also shown.

As expected, these four partitioning schemes generated quite different pictures of the total deformation density (examples obtained from $2 F_{1}$ and $3 F$ partitioning schemes are shown in Figure 4) because each fragmentation had to break different covalent bonds. This had a significant impact not

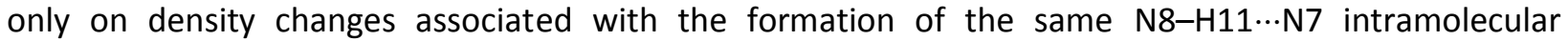
interaction, but also throughout a molecule - a full set of total deformation densities computed from four partitioning schemes is shown in Figure S1 in the Supplementary Information. Clearly, selecting a specific fragmentation scheme is not always a trivial exercise as it might be very impactful with regards to a final interpretation of energetic components, such as binding energy or Pauli, orbital and electrostatic terms of an interaction, computed from EDA. Most likely, from a visual inspection and analysis of energy terms computed, one would select the total deformation density generated from the $2 F_{1}$ scheme. This is because it resulted in the picture visualizing deformation densities within a region of the intramolecular interaction best when related to chemist's general knowledge - Figure 4(a). Such a heuristic approach is an easy choice for well-understood interactions, such as classical H-bonds, but is much more difficult to determine for unknown or controversial interactions, such as $\mathrm{CH} \cdots \mathrm{HC}$. Unavoidably, an additional and dominating contribution to the deformation density is observed which resulted from reconstruction of the covalent $\mathrm{C}-\mathrm{C}$ bond when two promolecules, $-\mathrm{CH}_{2} \mathrm{NH}_{2}$ and $-\mathrm{CH}_{2} \mathrm{NH}_{3}{ }^{+}$, were brought together from an infinite separation. 
It is clear from Figure 4 that the use of unphysical, promolecular reference states has an undesirable yet unavoidable effect on the intramolecular interaction of interest. The extent of this effect has been impossible to determine, casting doubt on any result obtained by EDA approaches for intramolecular interactions (including results based on the interaction energies). Clearly, it is desirable to obtain deformation densities for intramolecular interactions without using unphysical reference states, which is
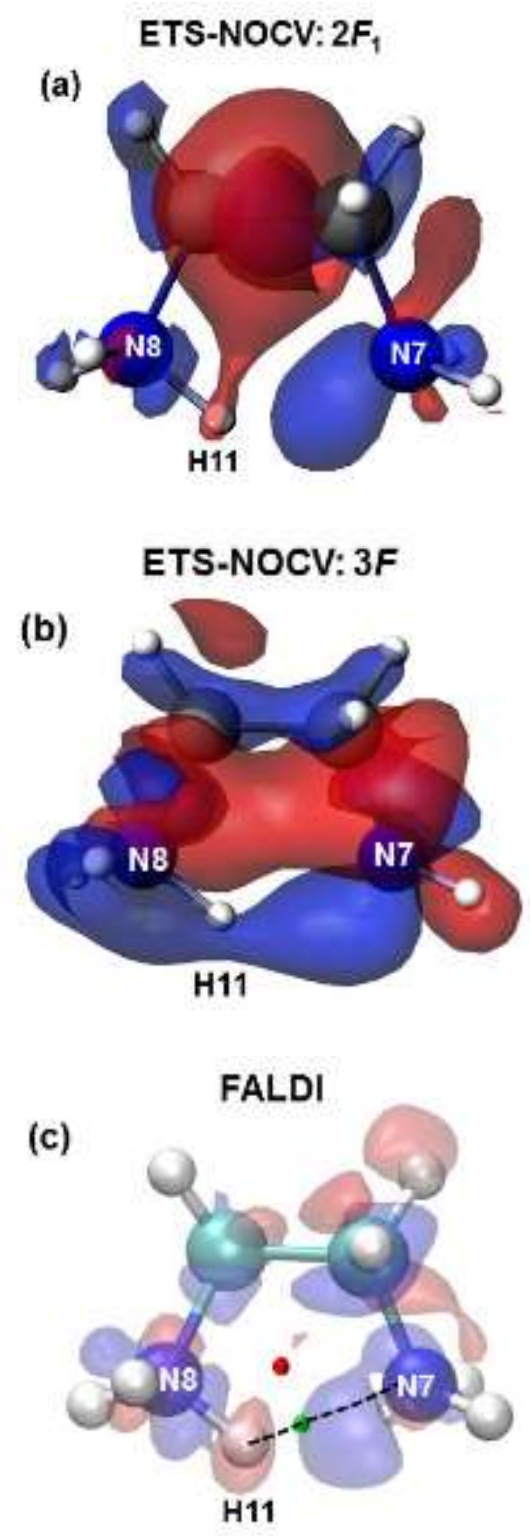

Figure 4. Total deformation densities: (a) and (b) computed in ADF using indicated partitioning scheme, (c) from FALDI, using the conformational approach. Red and blue regions indicate a decrease and increase in density, respectively, relative to the radical fragments in (a) and (b) and on the ref $\rightarrow$ fin structural change of $\mathrm{Hen}^{+}$in (c). All isosurfaces are at 0.0025 au. 
the primary justification for this work. To achieve such deformation densities, FALDI makes use of real, chemically viable reference states, here from a linear to equilibrium conformer of $\mathrm{Hen}^{+}-$Figure 3. A 3D isosurface for the total deformation density (TDD) resulting from the conformational FALDI approach is shown in Figure 4c. Comparing the two pictures in Figure 4a (EDA) and 4c (FALDI) reveals that there are similar features in the $\mathrm{H}_{2} \mathrm{~N} \cdots \mathrm{HNH}_{2}{ }^{+}$region, namely (i) an accumulation of density is observed for $\mathrm{N7}$ and N8 whereas $\mathrm{H} 11$ is losing density and (ii) polarization of terminal groups is observed which appears to be more significant for $-\mathrm{N} 7 \mathrm{H}_{2}$ from ETS-NOCV whereas FALDI points at $-\mathrm{N} 8 \mathrm{H}_{3}{ }^{+}$. However, the FALDIgenerated picture reveals additional regions that experienced a change in the density on a linear $\rightarrow$ equilibrium structural change. Importantly, these regions correspond to the atomic population changes well as, e.g., we obtained from QTAIM $\Delta N=+15,-8,-59$, and -21 me for $\mathrm{C} 1, \mathrm{C} 4, \mathrm{H} 2$, and H3, respectively (both $\mathrm{H}$-atoms of the $\mathrm{C}_{1} \mathrm{H}_{2}$ group). Such a picture cannot be recovered from an orthodox approach because it is largely distorted by the deformation density of a covalent bond formation.

To conclude our analysis of the intramolecular interaction region from the total deformation density perspective, it is clear that the FALDI-based approach displays similar features as those obtained from an orthodox approach, but without the problems caused by the use of unphysical reference states. Furthermore, FALDI density decomposition can also be applied on any number of fragments ge nerated in an orthodox fashion, by cutting a molecule to radical-state promolecules (at this stage, this is limited to wavefunctions generated by Gaussian). Although we are not going to extensively use the FALDI-onpromolecules approach in this work, it is important to stress that we were able to recover ADFgenerated images of the TDD using four partitioning schemes mentioned above; this is illustrated in Figures S2 in the Supplementary Information. This gives us an assurance that our approach is working well and no major bugs are present in the in-house developed software package. Finally, a full set of fragment deformation densities (FDD), calculated by the FALDI-on-promolecules approach, was obtained for the $-\mathrm{CH}_{2} \mathrm{CH}_{2}-$ and $\mathrm{H}_{2} \mathrm{~N} \cdots \mathrm{HNH}_{2}{ }^{+}$fragments. It is presented in Figure $\mathrm{S} 3$ and $\mathrm{S} 4$ in the Supplementary Information, showing how pictures recovered depend on a particular partition scheme used; for illustration purposes, FDDs obtained from $2 F_{1}$ and $3 F$ are shown in Figure 5 . Summing up FDDs from, e.g., the $2 \mathrm{~F}_{1}$ partitioning scheme, one obtains the TDD shown in Figure $4(\mathrm{a})$. This demonstrates that the FALDI decomposition can provide additional insight to orthodox deformation density studies by uncovering the origin of computed TDD. 


\section{FALDI-on-promolecules}
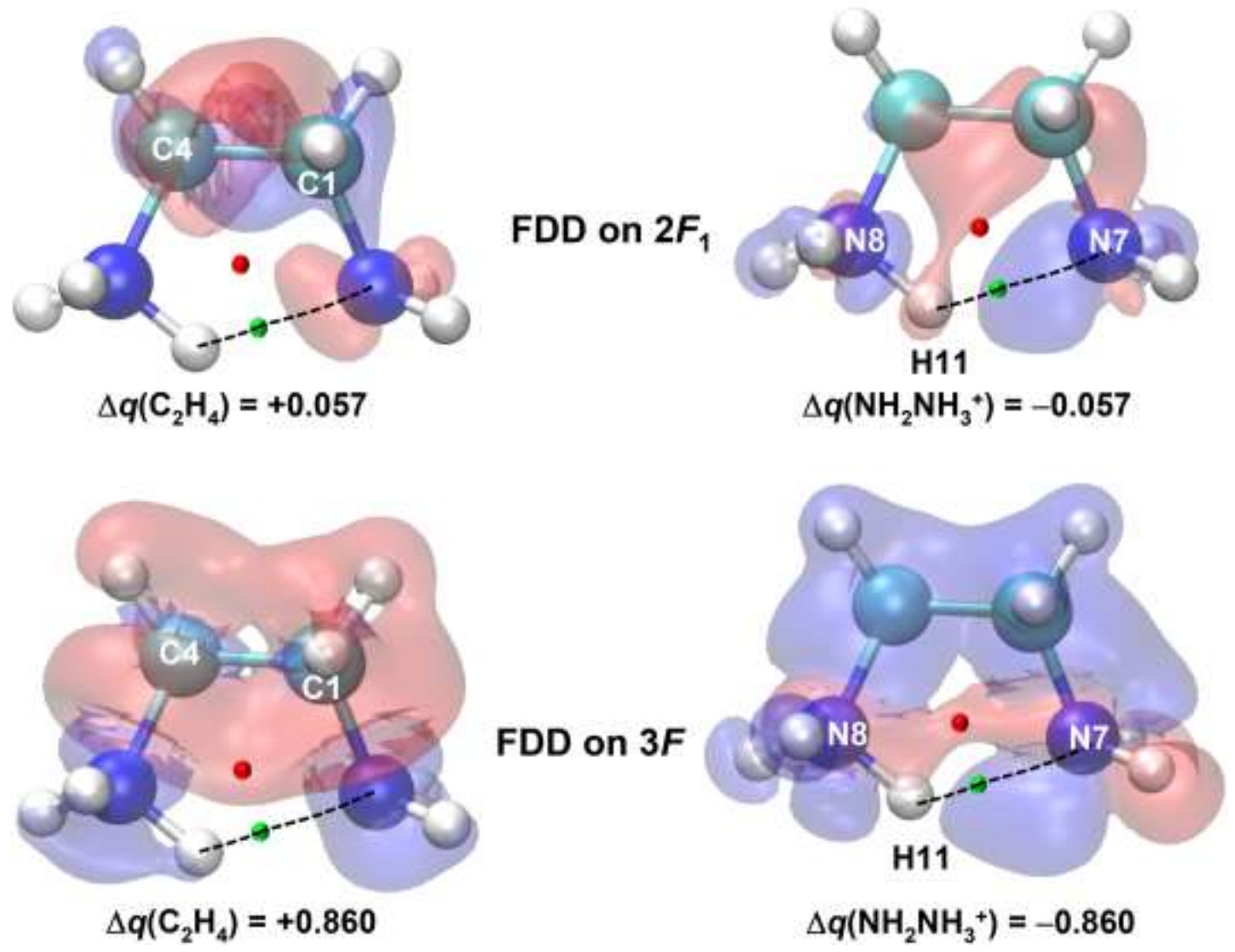

Figure 5. FALDI-on-promolecules generated FDD from the $2 F_{1}$ and $3 F$ partitioning schemes for $-\mathrm{C}_{4} \mathrm{H}_{2}-\mathrm{C}_{1} \mathrm{H}_{2}$-and combined terminal groups using orthodox promolecules as a reference state. All isosurfaces are at 0.0025 au.

4.2. Atomic FALDI deformation densities. It is important to realize that the total deformation density computed from the FALDI scheme, Figure 4(c), does not depict details related to individual atoms or functional groups. This is because the TDD is the sum of either atomic and/or fragments deformation densities. 


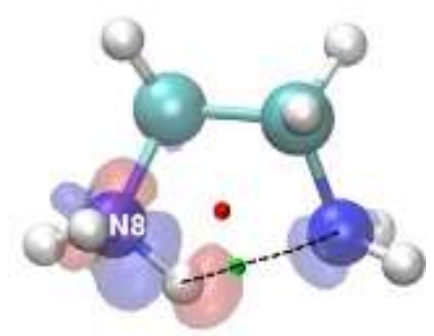

$\Lambda q(\mathbf{N} 8)=-0.025$
$\Delta N(N 8)=+0.025$

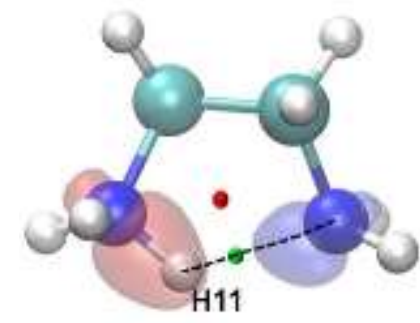

$\Delta q(\mathbf{H} 11)=+0.042$

$\Delta N(\mathrm{H} 11)=-0.042$

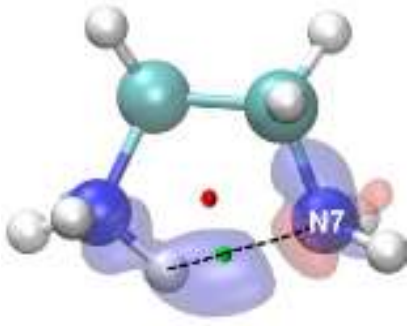

$\Delta q(N 7)=0.061$

$\Delta N(N 7)=+0.061$
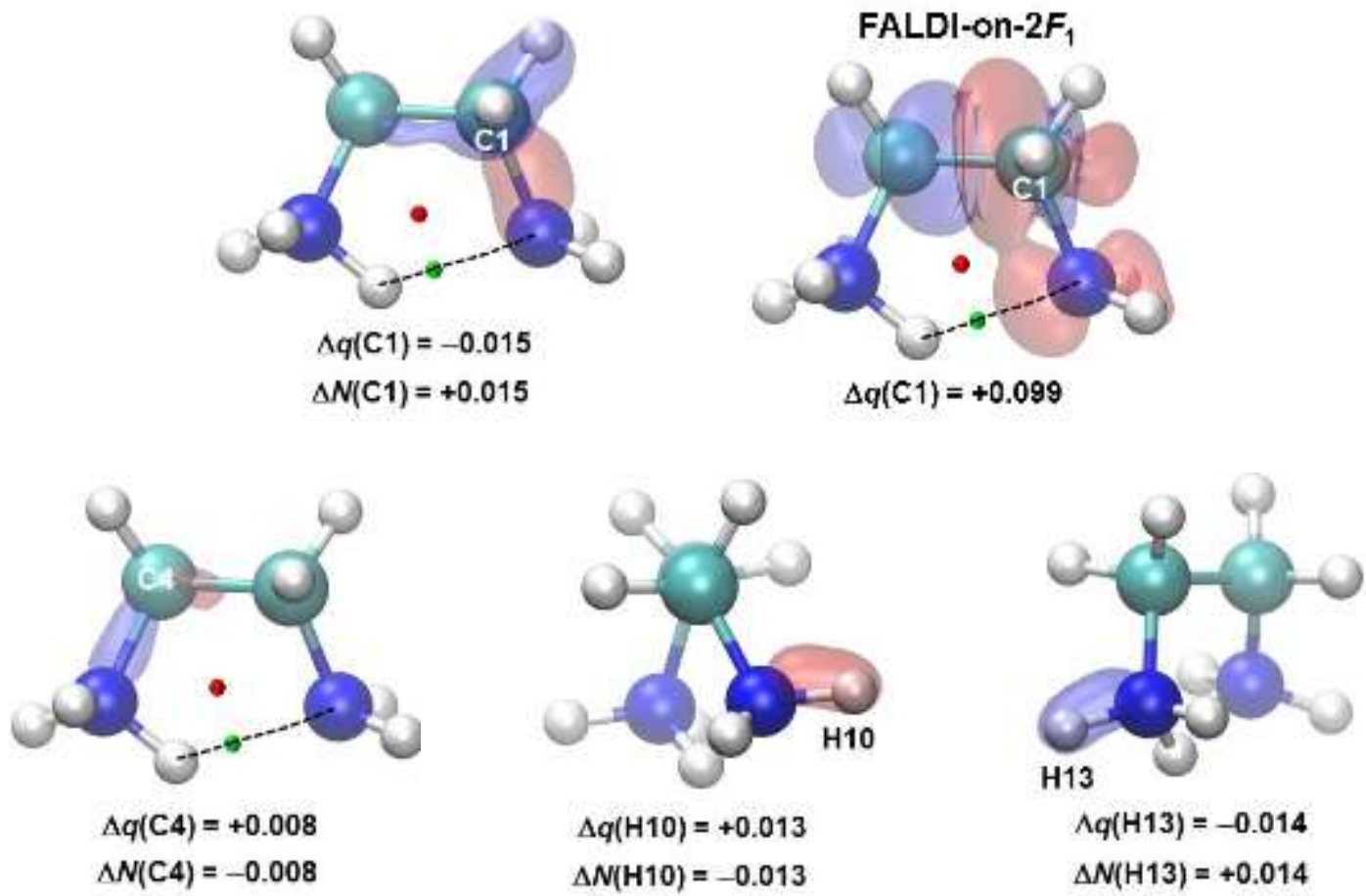

Figure 6. ADD distributions and changes in QTAIM atomic net charges and atomic populations (both in $e$ ) obtained for indicated atoms on the ref $\rightarrow$ fin structural change. Isosurfaces for $\mathrm{C} 4, \mathrm{H} 10$ and $\mathrm{H} 13$ are presented are at 0.001 au; all other isosurfaces are at 0.0025 au.

Hence, to uncover the origin of the conformational, from ref to fin state, deformation density in $\mathrm{Hen}^{+}$, in terms of individual atoms' contributions, we computed atomic deformation densities (ADDs) for each atom - a full set of FALDI-generated ADDs is shown in Figure S5 in the Supplementary Information, and for selected atoms in Figure 6.

According to the IUPAC recommendation, ${ }^{13}$ a wide range of $\mathrm{H}$-bonds should be represented as $\mathrm{X}-\mathrm{H} \cdots \mathrm{Y}-$ $\mathrm{Z}$, where $\mathrm{X}-\mathrm{H}$ represents the hydrogen bond donor and, in the case of an intramolecular $\mathrm{H}-$ bond, $\mathrm{Y}-\mathrm{Z}$ represents a molecular fragment acting as the acceptor, where $Y$ is bonded to $Z$. Moreover, a classical intramolecular $\mathrm{H}$-bond is commonly interpreted using the $\mathrm{X}-\mathrm{H} \cdots \mathrm{Y}$ notation when $\mathrm{H}$ is bonded to electronegative atom, $\mathrm{O}, \mathrm{N}$ or $\mathrm{F}^{58}$ Following this recommendations, we have analyzed $\mathrm{X}=\mathrm{N} 8, \mathrm{H}=\mathrm{H} 11, \mathrm{Y}$ $=\mathrm{N} 7$, and $\mathrm{Z}=\mathrm{C} 1$ as this selection affords us both notations and it was of great interest to find out if there are specific features of these atoms' ADDs (Figure 6) which could be linked with 4- and/or 3-atom representation. Comparing data shown in Figure S5 in the Supplementary Information reveals that by 
far, in terms of 3D spaces occupied, the most significant changes in density were indeed obtained for ADDs of $\mathrm{C} 1, \mathrm{~N} 7, \mathrm{H} 11$ and N8. As a matter of fact, these atoms are the only ones in $\mathrm{Hen}^{+}$for which the $A D D$ is delocalized towards more than one neighboring atom. This is indicative of their most important contributions made to the conformational TDD change and can be used as a textbook example in support of the IUPAC recommendation that also illustrates, in 3D space, atomic contributions on the formation of a classical intramolecular bond.

The nature and mechanism of a classical intermolecular $\mathrm{H}$-bonding, as $\mathrm{X}-\mathrm{H} \cdots \mathrm{Y}$, was studied extensively for years. It has been established from, e.g., EDA, ${ }^{30} \mathrm{DFT}^{59}$ and IQA studies ${ }^{60}$ that on formation of a $X-$ $\mathrm{H} \cdots \mathrm{Y}$ bond some charge transfer (CT) from $\mathrm{Y}$ (here N7) to $\mathrm{X}-\mathrm{H}$ (here N8-H11) always takes place. FALDI

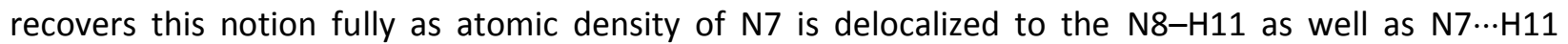
bonding region. In the latter case, density is placed close to $\mathrm{H} 11$ along the AlL which must facilitate formation of the AlL. However, we also see in Figure 6 that N7 is also delocalizing some density in the N7-C1 region and this is counteracted by $\mathrm{C} 1$ which is removing its density from this bonding region and placing it mainly in the $\mathrm{C} 1-\mathrm{H} 2$ interatomic region. A significant interplay in density redistribution between $\mathrm{N} 7$ and $\mathrm{C} 1$ in combination with large space occupied by C1's ADD further supports the 4-atom IUPAC notation.

An interesting picture is observed for N8 of the hydrogen bond donor. This atom is placing its density in the H11-N8 bonding region as well as on N7 (a proton acceptor) by removing it from $\mathrm{H} 11$ which is accompanied by charge redistribution, hence polarization of its own atomic basin. Furthermore, changes in density attributed to N8 near N7 and H11 are well separated and localized along the AlL.

It is known that $\mathrm{H}(\mathrm{H} 11)$ is losing charge and the FALDI-generated picture suggests that this atom's density is being delocalized by removing it not only from its own basin but also from the entire bonding region of the hydrogen bond donor $\mathrm{H} 11-\mathrm{N} 8$, as well as from the non-bonding region of N8. Noticeably, the removed density is placed entirely on the proton acceptor N7 and, importantly, it is located mainly

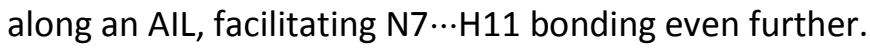

All these atomic density rearrangements provide the origin of polarization effects observed in Figure

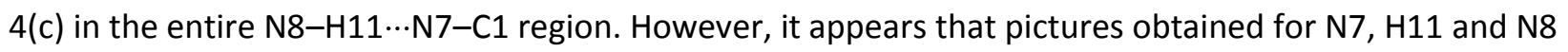
in Figure 6 also provide some support for the $\mathrm{X}-\mathrm{H} \cdots \mathrm{Y}$ representation of a classical $\mathrm{H}$-bonding. To this

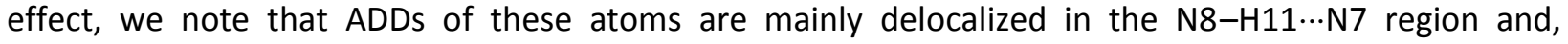
importantly, the two $\mathrm{N}$ atoms are the only ones which, not being neighbors, donated density through space to each other.

To complete the analysis of deformation densities computed for individual atoms, we would like to

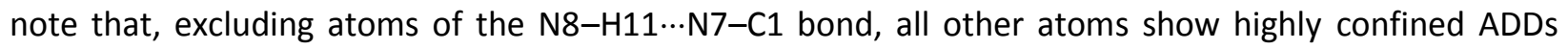
which are placed almost entirely along a single covalent bond. To illustrate this, examples for $\mathrm{C} 4$ (to compare it with $\mathrm{C} 1), \mathrm{H} 10$ and $\mathrm{H} 12$ of the $-\mathrm{NH}_{2}$ and $-\mathrm{NH}_{3}{ }^{+}$terminal groups, respectively, are shown in Figure 6. Finally, a 3D-ADD computed for $\mathrm{C} 1$ from the $2 F_{1}$ partitioning scheme, marked as FALDI on $2 F_{1}$, is also shown in Figure 6. It is immediately noticeable that FALDI-generated conformational ADD for C1, when the ref $\rightarrow$ fin structural change took place, is extremely different from that obtained using a FALDI-on-promolecules approach employing the $2 F_{1}$ partitioning scheme. Whereas FALDI shows that the $A D D$ is delocalized in the immediate vicinity of $C 1$ and neighboring atoms, totally unexpected, much larger and almost throughout a molecule ADD was obtained from the FALDI-on-promolecules approach. Such ADD must be seen as unphysical and nicely illustrates how reconstructing of a C-C covalent bond has a detrimental impact on the picture recovered, especially in terms of its impact on the intramolecualr $\mathrm{NH} \cdots \mathrm{N}$ interaction of interest. Furthermore, relative to the linear conformer of $\mathrm{Hen}^{+}$, 
deformation density of $\mathrm{C} 1$ from $2 F_{1}$, when the FALDI-on-promolecules approach was used, has resulted in the change of the net atomic charge, $\Delta \mathrm{q}(\mathrm{C} 1)=+0.099 e$ which is totally different in value and trend when compared with -0.015 e computed from QTAIM.

4.3. Fragment FALDI deformation densities. Atomic resolution implemented in the FALDI scheme provides an invaluable insight on atoms' involvement in bonding. However, formation of an intramolecular interaction can also be explored from the perspective of functional groups. As showed in Figure 4(c), formation of the intramolecular bond is a holistic, molecular wide event in terms of a charge redistribution and resultant polarisation effects. Hence, one would expect that the picture recovered using functional groups (FGs) should provide an additional and, to some extent, more general description. Atoms involved in $\mathrm{H}$-bonding are members of three $\mathrm{FGs}$ and they all were used as fragments in FALDI's scheme; 3D representation of FDDs attributed to the $-\mathrm{C} \mathrm{H}_{2}-,-\mathrm{N}^{-} \mathrm{H}_{2}$ and $-\mathrm{N} \mathrm{H}_{3}{ }^{+} \mathrm{FGs}$ is shown in Figure 7.

A comparative analysis of FDDs computed for the $-\mathrm{N} 7 \mathrm{H} 2$ and $-\mathrm{N} 8 \mathrm{H}_{3}{ }^{+}$functional groups (Figure 7) with ADDs of N7, H11 and N8 atoms in Figure 6 leads to the conclusions that there are similar, in principle identical in nature, features for both atomic and fragment resolution. This means that our interpretation of atomic deformation densities can be entirely extended to FDDs. This finding is of fundamental significance as it provides additional information on the origin of the TDD shown in Figure 4(c) by providing an evidence of a negligible contribution made by $\mathrm{H}$-atoms not directly involved in the bond formation ( $\mathrm{H} 9$ and $\mathrm{H} 10$ of $-\mathrm{N}_{7} \mathrm{H}_{2}, \mathrm{H} 12$ and $\mathrm{H} 13$ of $-\mathrm{N} 8 \mathrm{H}_{3}{ }^{+}$). The only difference is observed in the region of $\mathrm{H}$-atoms of the $-\mathrm{C} \mathrm{H}_{2}-$ fragment and the computed depletion of density

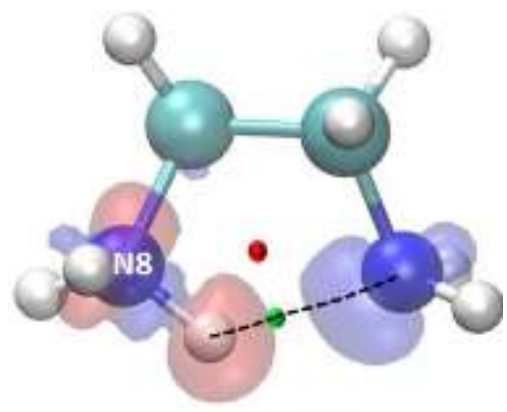

Fragment: $\mathrm{N}_{3} \mathrm{H}_{3}{ }^{+}$

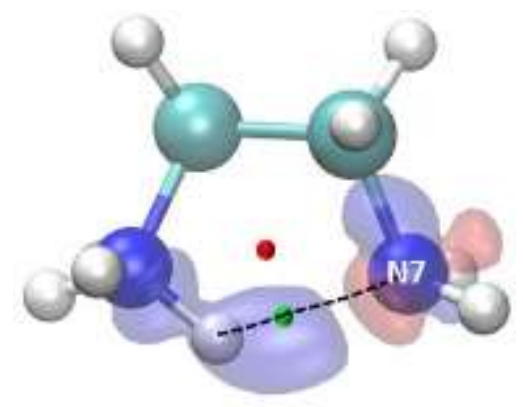

Fragment: $\mathrm{N}^{7} \mathrm{H}_{2}$

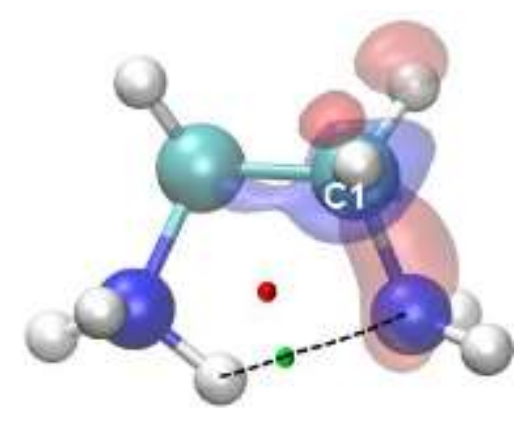

Fragment: $\mathrm{C}_{1} \mathrm{H}_{2}$

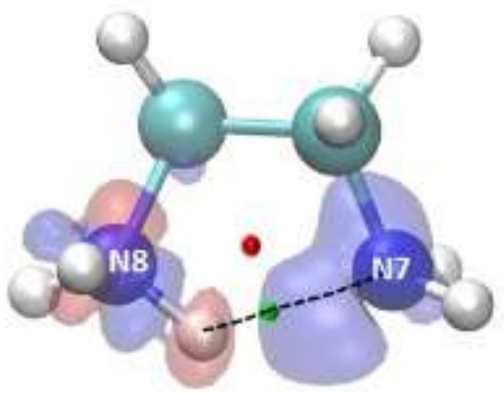

Fragment: $\mathrm{N}_{3} \mathrm{H}_{3}{ }^{+} \mathrm{N} 7 \mathrm{H}_{2}$

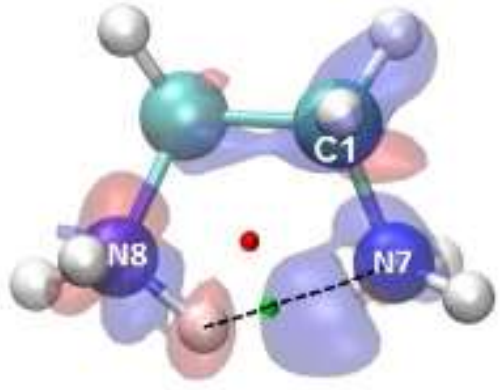

Fragment: $\mathrm{N}_{3} \mathrm{H}_{3}{ }^{+} \mathrm{N} 7 \mathrm{H}_{2} \mathrm{C} 1 \mathrm{H}_{2}$

Figure 7. FDD distributions obtained for indicated fragments on the $r e f \rightarrow f i n$ structural change. All isosurfaces are at 0.0025 au. 
correlates well with the largest decrease in the atomic population among all atoms of $\mathrm{Hen}^{+}$found for $\mathrm{H} 2$ whereas $\mathrm{H} 11$ experienced second largest outflow of density.

The combined deformation densities for two terminal groups, as $\mathrm{NH}_{2} \mathrm{NH}_{3}{ }^{+}$, produced a picture which is also consistent with a general description of a classical intermolecular $\mathrm{X}-\mathrm{H} \cdots \mathrm{Y}$ bond. We observe a combined charge redistribution which resulted in larger(smaller) charge accumulation on a proton accepter N7(donor N8) and density depletion on H11, a common feature also found from the IQA studies of a number of dimers using the full-valence CAS/ $6-311+G(d, p)$ wavefunction. ${ }^{60}$ Moreover, a general trend in the atomic population change, when the structure of $\mathrm{Hen}^{+}$changed from the linear to equilibrium conformer, $\Delta N(\mathrm{X})>|\Delta N(\mathrm{H})|>\Delta N(\mathrm{Y})$ obtained here at B3LYP/6-311++G $(\mathrm{d}, \mathrm{p})$ is the same when compared with that computed as a difference in atomic populations between monomers and dimers. ${ }^{60}$ This shows that general features of the charge redistribution, here nicely illustrated by the FDDs, and trends in computed atomic population changes are comparable and representative, regardless of level of theory used and the kind of classical $\mathrm{H}$-bonding, either inter- or intramolecular.

In conclusion, the atomic- and fragment-based analyses of deformation densities provided an invaluable fundamental insight on the mechanism of an interaction formation. Furthermore, ADDs and FDDs, by providing the origin, are extremely helpful in understanding and interpreting the overall picture obtained from the total deformation density. Regarding the two different representations $(X-H \cdots Y-Z$

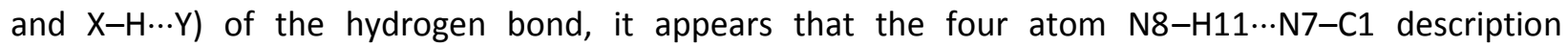
represents the intramolecular bonding better, because the ADD distribution for C1 (Figure 6) revealed that $\mathrm{C} 1$ also and significantly contributes to the density within the $\mathrm{NH} \cdots \mathrm{N}$ bonding region. Such a result cannot be obtained from a charge decomposition scheme, such as QTAIM, and is only revealed through FALDI's distributions in real space.

4.4. Diatomic and intrafragment interactions from the FALDI perspective. In the FALDI scheme, the decomposition of each delocalized deformation density (DDD) distribution into individual distributions of the changes in shared electrons across two or multiatomic basins results in the interaction deformation density (IDD) distributions. This is of special interest to us as it should provide a unique

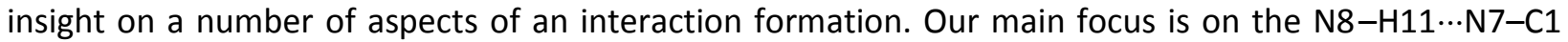
hydrogen bond (observed in the equilibrium structure of $\mathrm{Hen}^{+}$) which is characterized by the presence of Bader's AlL. Figure 8 displays IDD isosurfaces computed for four most relevant diatomic interactions, showing the real-space distribution of changes in QTAIM defined delocalization indices.

It is important to stress that in the IQA world all diatomic fragments are treated on equal footing and we wondered whether our analysis, based on interaction deformation densities, can show us unique features one could link with the formation, or an absence, of a BP. From a general inspection of isosurfaces in Figure 8 it is immediately seen that red(blue) isosurfaces representing a decrease(increase) in density in the interatomic region correlate perfectly well with the sign, negative(positive), of the delocalization indexes.

Because we analyze the outflow (red) and inflow (blue isosurface) of density from an interatomic region, it immediately appeals to the exchange-correlation (XC) term of the IQA-defined interatomic (or intrafragment) interaction energy. Looking at pictures recovered for the diatomic interaction in Figure 8 


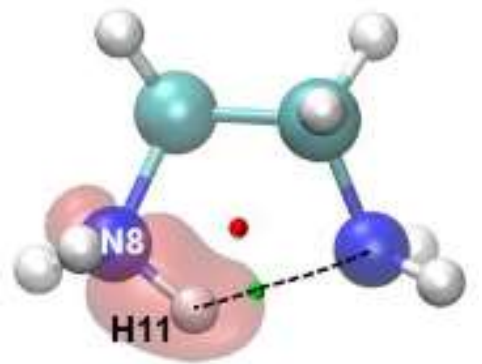

$\Delta \delta(\mathrm{N} 8, \mathrm{H} 11)=0.121$

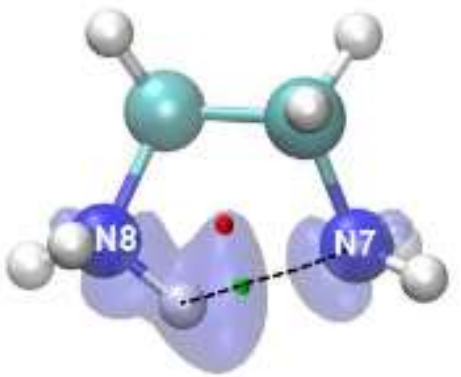

$\Delta \delta(N 8, N 7)=+0.075$

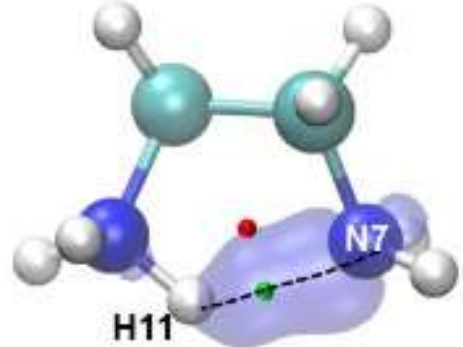

$\Delta \&(\mathrm{H} 11, \mathrm{~N} 7)=+0.118$

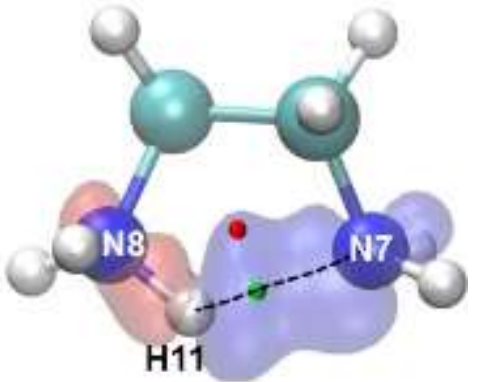

$\Delta \delta(\mathrm{N} 8, \mathrm{H} 11, \mathrm{~N} 7)=+0.072$

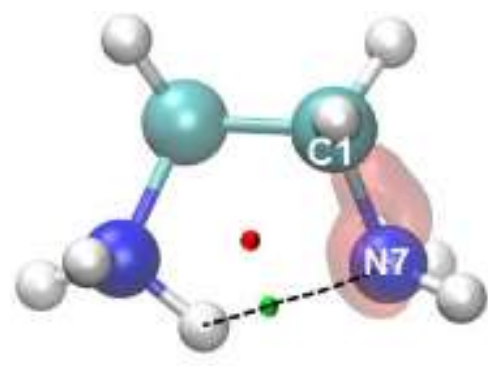

$\Delta \delta(C 1, N 7)=-0.038$

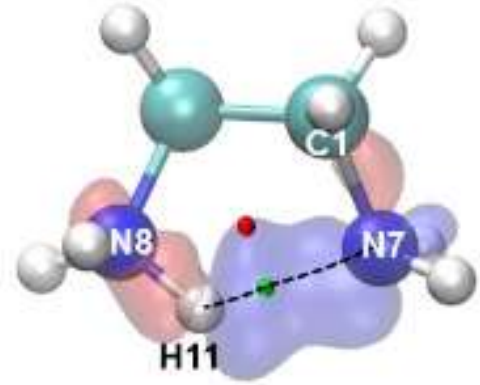

$\Delta \delta(N 8, H 11, N 7, C 1)=+0.013$

Figure 8. IDD distributions obtained for the indicated diatomic and intrafragment interactions. Red and blue regions indicate a decrease and increase in density, respectively, relative to the reference conformer. All isosurfaces are at 0.0025 au. Changes in QTAIM-defined delocalization indices are indicated.

recovered for the diatomic interaction in Figure 8 we note that the DI decreased for $\{\mathrm{C} 1, \mathrm{~N} 7\}$ and $\{\mathrm{N} 8, \mathrm{H} 11\}$ fragments with the latter correlating perfectly well with our general knowledge, and (ii) increased for the $\{\mathrm{N} 7, \mathrm{H} 11\}$ as well as $\{\mathrm{N} 7, \mathrm{~N} 8\}$ fragments, where the result obtained for the former corresponds to the formation of an intramolecular interaction in the form of Bader's BP. The above trends are fully supported by the computed $\mathrm{V}_{X C}^{A, B}$ terms for relevant diatomic interactions, as we obtained $\Delta \mathrm{V}_{X C}^{A, B}=+6.57$ and $+18.80 \mathrm{kcal} \cdot \mathrm{mol}^{-1}$ for $\{\mathrm{C} 1, \mathrm{~N} 7\}$ and $\{\mathrm{N} 8, \mathrm{H} 11\}$ interactions, and -16.59 and $8.02 \mathrm{kcal} \cdot \mathrm{mol}^{-1}$ for $\{\mathrm{N} 7, \mathrm{H} 11\}$ and $\{\mathrm{N} 7, \mathrm{~N} 8\}$ fragments, respectively. IDDs, however, allow for investigation of shared electrons throughout all space. Comparing IDDs for the $\{\mathrm{N} 7, \mathrm{H} 11\}$ and $\{\mathrm{N} 7, \mathrm{~N} 8\}$ fragments

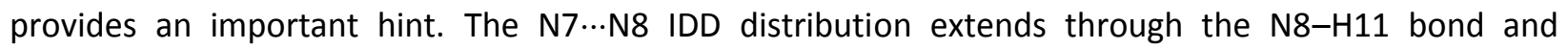
$\mathrm{N} 7 \cdots \mathrm{H} 11$ interaction. However, there is a fundamental difference between IDD distributions of $\{\mathrm{N} 7, \mathrm{H} 11\}$ and $\{N 7, N 8\}$ : whereas the former has a BP the latter does not have a channel-like feature of the N7 ‥H11 IDD distribution even though the density shared between N7 and N8 shows a large maximum exactly at the geometric middle point between the two atoms. In general, this correlates very well with density topology recovered from QTAIM and the concept of QTAIM-defined bond paths as privileged exchange channels proposed by Pendás et al. ${ }^{40}$ Importantly, it appears that defined here IDD distributions might provide a powerful tool to investigate the underlying delocalization patterns corresponding to a presence/absence of a BP in real-space. One must recall that an attempt of using the value of the delocalization indices came short in some instances in supporting (or explaining) a presence/absence of BP. ${ }^{61}$ It is our intention to explore this area in future studies. 


\section{Conclusions}

The simplicity and extreme usefulness of the general deformation density in revealing how charge is accumulated or depleted upon the formation of an intermolecular interaction is unfortunately marred by the inability of orthodox deformation densities to describe intramolecular interactions. The main limitation of any EDA scheme for the study of intramolecular interactions, namely a necessity of breaking existing bonds to generate arbitrarily selected fragments, has been addressed in this work by implementing Fragment, Atomic, Localized, Delocalized and Interatomic contributions that are computed within the novel FALDI charge decomposition scheme. The FALDI scheme is a result of a paradigm shift from analyzing molecular deformation density obtained from reconstructing a molecule from unphysical radical state fragments to computing decomposed density distributions in two conformational states (final and reference) of a molecule which are then used to generate atomic, fragment as well as total deformation densities resulting from the structural $r e f \rightarrow f i n$ transformation. To achieve that we have made use of few concepts originating from the Domain Averaged Fermi Hole $(\mathrm{DAFH})^{48,49}$ method and to account for different space occupied by the same atoms in the fin and ref states, a dedicated transformation scheme of atomic coordinates was implemented.

As such, the derived expressions for the atomic, fragment and total deformation densities can be used for any state of a molecule (giving static density distributions) without a need of its 'artificial' partitioning as well as they can be used on EDA-generated fragments. Importantly, in the latter case, which we call FALDI-on-promolecules approach, EDA-generated deformation densities are fully recovered providing validation of the protocol developed in this work. In principle, applying our approach on the same intramolecular interaction revealed a picture with similar features as found from the orthodox EDA approach. However, because FALDI eliminates the interference of the reconstructing of a nearby covalent bond, the conformational deformation density isosurfaces displayed a number of details involving the $\mathrm{H}$-bond which were either missing or masked when promolecular approaches are used.

As a case study, we used linear (as a ref state) and the lowest energy conformer (as a fin state) of protonated ethylenediamine, $\mathrm{Hen}^{+}$. The mechanism and origin of spontaneously formed intramolecular interaction in fin, $\mathrm{N}-\mathrm{H} \cdots \mathrm{N}$, was uncovered using deformation densities on atomic and molecular fragment levels. We have shown, for the first time in real space, how four atoms contribute to the resultant molecular deformation density on the interaction formation. Interestingly, these atoms' contributions can be used as a textbook example in support of the IUPAC recommendation, namely $X-$ $\mathrm{H} \cdots \mathrm{Y}-\mathrm{Z}(\mathrm{N}-\mathrm{H} \cdots \mathrm{N}-\mathrm{C}$ in the case studied here), in representing intramolecular $\mathrm{H}$-bonding. We also uncovered the influence of the $Z$ atom (here $\mathrm{C} 1$ ) on the density distribution in the bonding region of $\mathrm{H} \cdots \mathrm{N}$, a result which can only be obtained by visualizing delocalized density in real space. Furthermore, we also found unique features which explain the commonly used $\mathrm{N}-\mathrm{H} \cdots \mathrm{N}$ notation; the atomic

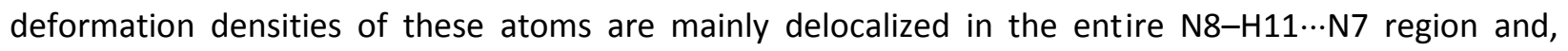
importantly, the two $\mathrm{N}$ atoms are the only ones which, not being neighbors, donated density through space to each other. A 3D picture of the deformation density computed for the N8-H11...N7 fragment nicely explains why a BP is only observed between the $\mathrm{H}$-atom and the proton acceptor $\mathrm{N}$-atom even though both $\mathrm{N}$-atoms delocalize their densities to each other. Whereas there is a continued increase in density in the bonding region of $\mathrm{H} 11 \cdots \mathrm{N} 7$, which is recovered by the presence of a BP between them, the interatomic region between $\mathrm{N}$-atoms not only does not show such a channel of increased density but also a resultant (final) depletion in density is observed close to N8-atom along the trajectory between $\mathrm{N} 8$ and N7. 
The added advantage of the FALDI scheme is in its ability of obtaining the total deformation from either atomic or/and fragment contributions. Such approach provides an invaluable insight on the origin and mechanism leading to or associated with the deformation density computed for an intramolecular interaction. This is of a fundamental significance as it should allow gaining an insight on the role played by a molecular environment (presence/absence of specific functionalities) in terms of density deformation leading to an intramolecular interaction. It is our conviction that FALDI might explain, on a fundamental level, the nature and origin of so many kinds of interactions. As a matter of fact, this is not limited to intramolecular ones as expressions derived can be equally used to understand all kinds of chemical bonds either in a final product or upon the formation of these bonds, hence providing an insight on a mechanism leading to density sharing. Furthermore, interatomic deformation densities (specifically defined within the FALDI scheme) should shed some light on the presence/absence of Bader's atomic interaction lines (or bond paths) that, in some instances, became a subject of heated debates when interpreted in terms of bonding or nonbonding character from a classical chemist's perspective. To this effect, the real-space nature of FALDI's delocalized density isosurfaces computed for the $\mathrm{N}-\mathrm{H} \cdots \mathrm{N}$ region corroborated fully with the interpretation of AlLs as privileged exchange channels ${ }^{40}$ and can provide significant support to this unorthodox interpretation; we are convinced that FALDI provides a promising method with which one should be able to elucidate the fundamentals behind the presence/absence of AlLs and their interpretations in terms of chemical bonding. Plotting interatomic delocalized density in real space also opens up additional avenues for studying concepts such as aromaticity, resonance and long-range atomic communication. Finally, we must stress that the FALDI decomposition also provides a strong density-based investigative tool for both deformation as well as static electron densities, which we hope will find use with both experimentalists as well as theoreticians.

\section{Acknowledgments}

This work is based on the research supported in part by the National Research Foundation of South Africa (Grant Number 87777) and the University of Pretoria.

Keywords: Deformation Density, FALDI, DAFH, Intramolecular Interactions, Computational chemistry

\section{Supporting Information.}

All Cartesian coordinates and electronic energies of structures used in this paper, partitioning schemes of a molecule, total deformation densities using orthodox fragmentation methods, total deformation densities using the FALDI-on-promolecules approach for all fragmentation schemes, selected fragment deformation densities using the FALDI-on-promolecules approach, full set of FALDI's atomic deformation densities. This material is available free of charge via the Internet at http://pubs.acs.org.

\section{References and Notes}

1. J. J. Novoa, M-H. Whangbo, J. M. Williams, J. Chem. Phys. 1991, 94, 4835-4841 
2. K. N. Robertson, O. Knop, T. S. Cameron, Can. J. Chem. 2003, 81, 727-743

3. R. H. Crabtree, Science 1998, 282, 2000-2001

4. R. Custelcean, J. E. Jackson, Chem. Rev. 2001, 101, 1963-1980

5. M. J. Calhorda, Chem. Commun. 2000, 801-809

6. T. B. Richardson, S. de Gala, R. H. Crabtree, P. E. M. Siegbahn, J. Am. Chem. Soc. 1995, 117, 1287512876

7. R. H. Crabtree, P. E. M. Siegbahn, O. Eisenstein, A. L. Rheingold, Acc. Chem. Res. 1996, 29, 348-354

8. S. J. Grabowski, W. A. Sokalski, J. Leszczynksi, Chem. Phys. 2007, 337, 68-76

9. U. Koch, P. L. A. Popelier, J. Phys. Chem. 1995, 99, 9747-9754

10. P. Hobza, Z. Havlas, Chem. Rev. 2000, 100, 4253-4264

11. I. Akorta, I. Rozas, J. Elguero, Chem. Soc. Rev., 1998, 27, 163-170

12. M. J. Calhorda, Chem. Commun. 2000, 10, 801-809

13. E. Arunan, G. R. Desiraju, R. A. Klein, J. Sadlej, S. Scheiner, I. Alkorta, D. C. Clary, R. H. Crabtree, J. J. Dannenberg, P. Hobza, H. G. Kjaergaard, A. C. Legon, B. Mennucci, D. J. Nesbitt, Pure Appl. Chem. 2011, 83, 1637-1641

14. P. Politzer, P. Lane, M. C. Concha, Y. Ma, J. S. Murray, Mol. Model 2007, 13, 305-311

15. S. Kawai, A. Sadeghi, F. Xu, L. Peng, A. Orita, J. Otera, S. Goedecker, E. Meyer, ACS Nano 2015, 9, 2574-2583

16. G. R. Desiraju, P. S. Ho, L. Kloo, A. C. Legon, R. Marquardt, P. Metrangolo, P. Politzer, G. Resnati, K. Rissanen, Pure Appl. Chem. 2013, 85, 1711-1713

17. F. Groenewald, C. Esterhuysen, J. Dillen, Theor. Chem. Acc. 2012, 131, 1-12

18. W. Wang, B. Ji, Y. Zhang, J. Phys. Chem. A 2009, 113, 8132-8135

19. E. A. Zhurova, C. F. Matta, N. Wu, V. V. Zhurov, A. A. Pinkerton, J. Am. Chem. Soc. 2006, 128, 8849-8861

20. C. F. Matta, J. Hernández-Trujillo, T. H. Tang, R. F. W. Bader, Chem. Eur. J. 2003, 9, 1940-1951

21. R. F. W. Bader, J. Phys. Chem. A 2009, 113, 10391-10396

22. A. M. Pendás, E. Francisco, M. A. Blanco, C. Gatti, Chem. Eur. J. 2007, 13, 9362-9371

23. F. Cortés-Guzmán, J. Hernández-Trujillo, G. Cuevas, J. Phys. Chem. A 2003, 107, 9253-9256

24. J. Echeverría, G. Aullón, D. Danovich, S. Shaik, S. Alvarez, Nat. Chem. 2011, 3, 323-330;

25. J. Poater, M. Solà, F. M. Bickelhaupt, Chem. Eur. J. 2006, 12, 2889-2895

26. J. Poater, M. Solà, F. M. Bickelhaupt, Chem. Eur. J. 2006, 12, 2902-2905.

27. P. Dem'yanov, P. Polestshuk, Chem. Eur. J. 2012, 18, 4982-4993.

28. The Chemical Bond: fundamental aspects of chemical bonding, G. Frenking, S. Shaik, Eds.; Wiley: New York, 2014; Vol. 1

29. I. Cukrowski, Comp. Theory Chem. 2015, 1066, 62-75

30. K. Kitaura, K. Morokuma, Int. J. Quantum Chem. 1976, 10, 325-340

31. T. Ziegler, A. Rauk, Theoretica chimica acta, 1977, 46, 1-10

32. M. A. Blanco, A. M. Pendás, E. Francisco, J. Chem. Theory Comp. 2005, 1, 1096-1109

33. R. Moszynski, Mol. Phys. 1996, 88, 741-758

34. R. F. W. Bader, in Atoms in Molecules: A Quantum Theory; Oxford University Press: Oxford, 1990.

35. K. Fukui, T. Yonezawa, H. Shingu, J. Chem. Phys. 1952, 20, 722-725

36. A. E. Reed, F. Weinhold, J. Chem. Phys. 1985, 83, 1736-1740

37. E. R. Johnson, S. Keinan, P. Mori-Sanchez, J. Contreras-Garcia, A. J. Cohen, W. Yang, J. Am. Chem. Soc. 2010, 132, 6498-6506

38. A. Michalak, M. Mitoraj, T. Ziegler, J. Phys. Chem. A 2008, 112, 1933-1939

39. M. P. Mitoraj, A. Michalak, T. Ziegler, J. Chem. Theory Comp. 2009, 5, 962-975

40. A. M. Pendás, E. Francisco, M. A. Blanco, C. Gatti, Chem. Eur. J. 2007, 13, 9362-9371

41. C. Foroutan-Nejad, S. Shahbazian, R. Marek, Chem. Eur. J. 2014, 20, 10140-10152. 
42. R. P. Feynman, Phys. Rev. 1939, 56, 340-344

43. I. Cukrowski, J. H. de Lange, A. S. Adeyinka, P. Mangondo, Comp. Theo. Chem. 2015, 1053, 60-76

44. I. Cukrowski, P. Mangondo, J. Comp. Chem. 2016, 37, 1373-1387

45. I. Cukrowski, K. K. Govender, M. P. Mitoraj, M. Srebo, J. Phys. Chem. A 2011, 115, 12746-12757

46. I. Cukrowski, J. H. de Lange, M. P. Mitoraj, J. Phys. Chem. A 2014, 118, 623-637

47. R. F. W. Bader, Chem. Eur. J. 2006, 12, 2896-2901

48. R. Ponec, J. Math. Chem. 1997, 21, 323-333

49. R. Ponec, J. Math. Chem. 1998, 23, 85-103

50. R. McWeeny, Rev. Mod. Phys. 1960, 32, 335-369]

51. E. Francisco, A. M. Pendás, A. Costales, Phys. Chem. Chem. Phys. 2014, 14, 4586-4597

52. P. Bultinck, D. L. Cooper, R. Ponec, J. Phys. Chem. A 2010, 114, 8754-8763

53. A. M. K. Müller, Phys. Lett. A 1984, 105, 446-452

54. Gaussian 09, Revision D.01, M. J. Frisch, G. W. Trucks, H. B. Schlegel, G. E. Scuseria, M. A. Robb, J. R. Cheeseman, G. Scalmani, V. Barone, B. Mennucci, G. A. Petersson, H. Nakatsuji, M. Caricato, X. Li, H. P. Hratchian, A. F. Izmaylov, J. Bloino, G. Zheng, J. L. Sonnenberg, M. Hada, M. Ehara, K. Toyota, R. Fukuda, J. Hasegawa, M. Ishida, T. Nakajima, Y. Honda, O. Kitao, H. Nakai, T. Vreven, J. A., Jr. Montgomery, J. E. Peralta, F. Ogliaro, M. Bearpark, J. J. Heyd, E. Brothers, K. N. Kudin, V. N. Staroverov, R. Kobayashi, J. Normand, K. Raghavachari, A. Rendell, J. C. Burant, S. S. Iyengar, J. Tomasi, M. Cossi, N. Rega, J. M. Millam, M. Klene, J. E. Knox, J. B. Cross, V. Bakken, C. Adamo, J. Jaramillo, R. Gomperts, R. E. Stratmann, O. Yazyev, A. J. Austin, R. Cammi, C. Pomelli, J. W. Ochterski, R. L. Martin, K. Morokuma, V. G. Zakrzewski, G. A. Voth, P. Salvador, J. J. Dannenberg, S. Dapprich, A. D. Daniels, Ö. Farkas, J. B. Foresman, J. V. Ortiz, J. Cioslowski, D. J. Fox, Gaussian, Inc., Wallingford CT, 2009.

55. AIMAll (Version 16.05.18), T. A. Keith, TK Gristmill Software, Overland Park KS, USA, 2016 (aim.tkgristmill.com)

56. W. Humphrey, A. Dalke, K. Schulten, J. Molec. Graphics 1996, 14, 33-38

57. ADF2014, SCM, Theoretical Chemistry, Vrije Universiteit, Amsterdam, The Netherlands,http://www.scm.com. E. J. Baerends, T. Ziegler, A. J. Atkins, J. Autschbach, D. Bashford, A. Bérces, F. M. Bickelhaupt, C. Bo, P. M. Boerrigter, L. Cavallo, D. P. Chong, D. V. Chulhai, L. Deng, R. M. Dickson, J. M. Dieterich, D. E. Ellis, M. van Faassen, L. Fan, T. H. Fischer, C. Fonseca Guerra, M. Franchini, A. Ghysels, A. Giammona, S. J. A. van Gisbergen, A. W. Götz, J. A. Groeneveld, O. V. Gritsenko, M. Grüning, S. Gusarov, F. E. Harris, P. van den Hoek, C. R. Jacob, H. Jacobsen, L. Jensen, J. W. Kaminski, G. van Kessel, F. Kootstra, A. Kovalenko, M. V. Krykunov, E. van Lenthe, D. A. McCormack, A. Michalak, M. Mitoraj, S. M. Morton, J. Neugebauer, V. P. Nicu, L. Noodleman, V. P. Osinga, S. Patchkovskii, M. Pavanello, C. A. Peeples, P. H. T. Philipsen, D. Post, C. C. Pye, W. Ravenek, J. I. Rodríguez, P. Ros, R. Rüger, P. R. T. Schipper, H. van Schoot, G. Schreckenbach, J. S. Seldenthuis, M. Seth, J. G. Snijders, M. Solà, M. Swart, D. Swerhone, G. te Velde, P. Vernooijs, L. Versluis, L. Visscher, O. Visser, F. Wang, T. A. Wesolowski, E. M. van Wezenbeek, G. Wiesenekker, S. K. Wolff, T. K. Woo, A. L. Yakovlev

58. Hydrogen bonding: new insights, Grabowski, S. J., Ed.; Springer: The Netherlands 2006; Vol. 3

59. O. Gálvez, P. C. Gómez, L. F. Pacios, J. Chem. Phys. 2001, 115, 11166-11184

60. A. M. Pendás, M. A. Blanco, E. Francisco, J. Chem. Phys. 2006, 125, 184112-184120

61. V. Tognetti, L. Joubert, J. Chem. Phys. 2013, 138, 024102-024109 
GRAPHICAL ABSTRACT

JURGENS DE LANGE, IGNACY CUKROWSKI

TOWARDS DEFORMATION DENSITIES FOR INTRAMOLECULAR INTERACTIONS WITHOUT UNPHYSICAL REFERENCE STATES USING FALDI-DD

A novel methodology for calculating deformation densities for intramolecular interactions without the need for unphysical (radical) fragments. A density decomposition scheme is introduced (FALDI) which is used to calculate and transform the density contributions of atoms, fragments and interatomic interactions of a molecule undergoing conformational change. The FALDI scheme also provides visualization of atomic and interatomic density in real-space, and is a useful analysis tool applicable on static electron densities, conformational and fragment-based deformation densities.

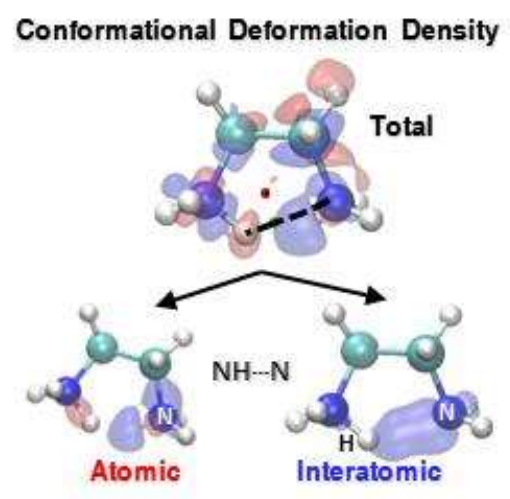

